\title{
ARTIN'S CONJECTURE, TURING'S METHOD AND THE RIEMANN HYPOTHESIS
}

\author{
ANDREW R. BOOKER
}

\begin{abstract}
We present a group-theoretic criterion under which one may verify the Artin conjecture for some (non-monomial) Galois representations, up to finite height in the complex plane. In particular, the criterion applies to $S_{5}$ and $A_{5}$ representations. Under more general conditions, the technique allows for the possibility of verifying the Riemann hypothesis for Dedekind zeta functions of non-abelian extensions of $\mathbb{Q}$.

In addition, we discuss two methods for locating zeros of arbitrary $L$-functions. The first uses the explicit formula and techniques developed in BS05 for computing with trace formulae. The second method generalizes that of Turing for verifying the Riemann hypothesis. In order to apply it we develop a rigorous algorithm for computing general $L$-functions on the critical line via the Fast Fourier Transform.

Finally, we present some numerical results testing Artin's conjecture for $S_{5}$ representations, and the Riemann hypothesis for Dedekind zeta functions of $S_{5}$ and $A_{5}$ fields.
\end{abstract}

\section{INTRODUCTION}

1.1. Artin's conjecture. Let $K / \mathbb{Q}$ be a Galois extension and $\rho: \operatorname{Gal}(K / \mathbb{Q}) \rightarrow \mathrm{GL}_{n}(\mathbb{C})$ a non-trivial, irreducible representation of its Galois group. In Art30, Artin associated to this data an $L$-function $L(s, \rho)$, defined initially for $\Re(s)>1$, which he conjectured to continue to an entire function and satisfy a functional equation. By a theorem of Brauer Bra47, one now knows the meromorphic continuation and functional equation of Artin's $L$-functions. The question remains whether they can have poles in the critical strip $0<\Re(s)<1$.

Artin established his conjecture for the monomial representations, those induced from a 1-dimensional representation of a subgroup; this of course includes all 1-dimensional $\rho$, in which case $L(s, \rho)=L(s, \chi)$ for a Dirichlet character $\chi$. Although the conjecture has not been decided in any dimension $\geq 2$, more evidence is provided in dimension 2 by the Langlands-Tunnell theorem [Lan80, Tun81, which affirms the conjecture for those representations whose image in $\mathrm{PGL}_{2}(\mathbb{C})$ is isomorphic to $A_{4}$ (tetrahedral) or $S_{4}$ (octahedral); only the $A_{5}$ (icosahedral) case remains. When $\rho$ is an odd icosahedral representation, meaning $\operatorname{det} \rho$ determines an odd Dirichlet character, infinitely many examples of Artin's conjecture are known from the work of Taylor et al. BBDBT01, Tay03.

Moreover, in the odd 2-dimensional case, there is an algorithm for verifying the conjecture, as follows. By a construction of Deligne and Serre DS74, given a holomorphic

The author was supported by an NSF postdoctoral fellowship. 
modular form $f$ of weight 1 , one may associate an odd 2-dimensional representation $\rho$ such that $L(s, f)=L(s, \rho)$. Conversely, every odd 2-dimensional $\rho$ such that $L(s, \rho)$ is entire arises from the Deligne-Serre construction. For any particular $\rho$, one can search for the associated form; once found, comparing the representation constructed by Deligne-Serre to $\rho$ via an effective version of the Cebotarev density theorem allows one to deduce the conjecture for $\rho$. This and other related techniques have been carried out in a number of cases; see Buh78, Kim94, JM00, BS02.

On the other hand, if one considers even 2-dimensional representations, the situation is somewhat different. There as well the conjecture has been established for all but the icosahedral cases. However, the correspondence is not with holomorphic forms, but rather Maass forms of eigenvalue $\frac{1}{4}$. Unfortunately, no analogue of the result of Deligne and Serre is known in that setting. Moreover, computation of the associated forms remains elusive; existing techniques (see e.g. BSV05]) only allow one to calculate Maass forms to within a prescribed precision, never exactly. Thus, at present this approach does not yield an algorithm for verifying Artin's conjecture.

The apparent difference between these two cases leads naturally to the following question: Given a Galois representation $\rho$, is there an algorithm that will decide in finite time, with proof, whether $L(s, \rho)$ is entire? Note that like the Riemann hypothesis, Artin's conjecture is falsifiable, i.e. it may be disproven by observing a counterexample, in this case a pole. The challenge is thus to find a way of demonstrating the conjecture when true.

Although we are unable to provide a definitive answer to this question, one approach, at least for 2-dimensional representations, is suggested by a theorem from [Boo03b]: If a given 2-dimensional $\rho$ is not associated to a holomorphic or Maass form as above, then $L(s, \rho)$ has infinitely many poles. In particular, once $L(s, \rho)$ has at least one pole, it must have infinitely many. Unfortunately, the result is ineffective, in the sense that it does not predict where the first pole must occur. A natural question, therefore, is whether an effective version of this theorem exists. First, however, we must consider exactly what that would mean; since the only handle that we have on an Artin $L$-function in the critical strip is as the ratio of entire functions given by Brauer's theorem, it is not immediately clear that we can check its holomorphy at a zero of the denominator without a priori knowing a lower bound on the residue of any poles.

In this paper we address precisely this issue, in Section 2 . There we present a criterion which, when satisfied, yields an algorithm for verifying the holomorphy of an Artin $L$ function up to a given height in the critical strip. In particular, we give the first direct evidence (as far as we are aware) of holomorphy in the critical strip of an $L$-function for which the conjecture cannot be established through the methods mentioned above. Although our criterion is not always satisfied, we are in general able to deduce partial information, such as a bound on the multiplicities and residues of possible poles. Moreover, the limitations of the information that we obtain give an idea of the hypotheses that one would have to impose in any effective version of the converse theorem in order to make the above approach work. 
1.2. Turing's method and the Riemann hypothesis. One application of our criterion is to the Riemann hypothesis for Dedekind zeta functions. Turing [Tur53 devised a method for checking the hypothesis in a bounded region for the Riemann $\zeta$ function. ${ }^{1}$ The method depends on the simplicity of the zeros of $\zeta$. Because of that, it is only directly extendable to Dedekind zeta functions of non-normal extensions of small degree (see [Tol97]) or abelian extensions, for which it is more natural to verify the hypothesis for the associated Dirichlet $L$-functions instead (see [Rum93]).

Similarly, for a non-abelian extension one can factorize the zeta function into Artin $L$ functions of irreducible representations. As these are also expected to have simple zeros, Turing's method applies, provided one assumes the Artin conjecture. However, combining our criterion with Turing's method, we will in some cases be able to deduce the Riemann hypothesis and holomorphy of the relevant Artin $L$-functions simultaneously. In fact, as we will see, there are even cases where we may check the Riemann hypothesis without being able to verify Artin's conjecture. We carry out the necessary generalization of Turing's method in Section 4 .

1.3. Rigorous zero computations. In order to implement these ideas, we develop, in Sections 3 and 5 , two methods of locating zeros of $L$-functions. The first uses the explicit formula and techniques developed for the Selberg trace formula in BS05. If one assumes the Riemann hypothesis, this method may be used with our criterion, in place of Turing's method, for verifying the Artin conjecture. More importantly, the explicit formula is clean to implement and yields estimates for low zeros quickly. It can thus serve as a check for later computations, or to fine tune the parameters of Turing's method for greater speed.

The second method is a technique for fast, rigorous computations of $L$-functions on the critical line. This is a hard problem in general, basically because of the difficulty of providing uniform, effective bounds for the relevant Mellin transforms. By making use of the Fast Fourier Transform, our technique allows one to compute many values of the same $L$-function simultaneously, which is particularly appropriate for Turing's method. In doing so, we need only consider a single Mellin transform, making rigorous computation more practical. In addition, the method has complexity comparable to that of computing a single value by the approximate functional equation.

Although our primary interest is in Artin $L$-functions, we carry out the details of Sections 3, 4 and 5 for arbitrary $L$-functions $L(s)$, in the hope that the results may be useful outside of the present context. More precisely, we make the following assumptions, notations and conventions throughout:

- $L(s)$ is given by an Euler product of degree $r$ :

$$
L(s)=\prod_{p \text { prime }} \frac{1}{\left(1-\alpha_{p, 1} p^{-s}\right) \cdots\left(1-\alpha_{p, r} p^{-s}\right)},
$$

\footnotetext{
${ }^{1}$ Reading Turing's paper on the subject, which was his last, one marvels at what he accomplished with the limited computational resources of the day. His method was truly ahead of its time.
} 
where the $\alpha_{p, j}$ are complex parameters satisfying the individual bound $\left|\alpha_{p, j}\right| \leq p^{1 / 2}$, and the product is absolutely convergent for $\Re(s)>1$. Further, for all but finitely many $p$, there is a pairing $\alpha \mapsto \alpha^{\prime}$ such that $\left|\alpha_{p, j} \alpha_{p, j}^{\prime}\right|=1$. For the exceptional $p$, such a pairing exists for a subset of the $\alpha_{p, j}$, and those not in the subset satisfy $\left|\alpha_{p, j}\right| \leq 1$

- Define

$\Gamma_{\mathbb{R}}(s):=\pi^{-s / 2} \Gamma\left(\frac{s}{2}\right), \quad \gamma(s):=\epsilon N^{\frac{1}{2}\left(s-\frac{1}{2}\right)} \prod_{j=1}^{r} \Gamma_{\mathbb{R}}\left(s+\mu_{j}\right), \quad \Lambda(s):=\gamma(s) L(s)$,

where $|\epsilon|=1, N$ is a positive integer and $\Re\left(\mu_{j}\right) \geq-\frac{1}{2}$. For a certain choice of these

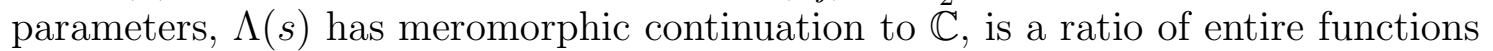
of order 1 , and satisfies the functional equation

$$
\Lambda(s)=\bar{\Lambda}(1-s),
$$

where for a complex function $f$ we denote by $\bar{f}(s)$ the function $\overline{f(\bar{s})}$. Note that $\epsilon$ here is the square root of the usual root number, and is only defined up to multiplication by \pm 1 ; we choose the value with argument in $\left(-\frac{\pi}{2}, \frac{\pi}{2}\right]$. Including $\epsilon$ as part of the $\gamma$ factor makes $\Lambda(s)$ real for $\Re(s)=\frac{1}{2}$, as can be seen from (1.3).

Let $Q(s)$ be the analytic conductor:

$$
Q(s):=N \prod_{j=1}^{r} \frac{s+\mu_{j}}{2 \pi} .
$$

Note that $\gamma(s)$ satisfies the recurrence $\gamma(s+2)=Q(s) \gamma(s)$. Further, we define

$$
\chi(s):=\frac{\bar{\gamma}(1-s)}{\gamma(s)}
$$

so that $L(s)=\chi(s) \bar{L}(1-s)$.

- $L(s)$ may have at most finitely many poles, which we assume to lie along the line $\Re(s)=1$. We label them $1+\lambda_{k}$ with $\lambda_{k} \in i \mathbb{R}, k=1, \ldots, m$, repeating with the appropriate multiplicity. Further, from the functional equation (1.3), each $\lambda_{k}$ will equal $-\mu_{j}$ for some $j$, counting multiplicity; in particular, $m \leq r$. We set

$$
P(s):=\prod_{k=1}^{m}\left(s-\lambda_{k}\right)
$$

so that $P(s) P(s-1) \Lambda(s)$ is entire.

- Some progress is known toward the Ramanujan conjecture for $L$; that is, there exists $\theta<\frac{1}{2}$ such that

$$
\left|\alpha_{p, j}\right| \leq p^{\theta} \quad \text { and } \quad \Re\left(\mu_{j}\right) \geq-\theta
$$

for all $p, j$. This assumption is not strictly necessary, as we could instead use average bounds of Rankin-Selberg type. However, bounds of the form (1.7) are now known 
in the cases of greatest interest (automorphic $L$-functions LRS99]), and the results are easier to state and use assuming it.

1.4. Numerical results. Finally, in Section 6 we describe the implementation of the above ideas and give some numerical results of tests of the Riemann hypothesis for a few $S_{5}$ and $A_{5}$ extensions in the region $|\Im(s)| \leq 100$. For the $S_{5}$ cases, this includes a verification of Artin's conjecture in the same region for the $L$-functions of all representations of the group.

\section{ACKNOWLEDGEMENTS}

The idea for this paper arose from a conversation with Laurent Clozel, and it is a pleasure to acknowledge him here. I thank Harold Stark, who has considered problems of this nature in the past and whose interest motivated me to finish the project. Thanks also to Bob Griess, Martin Isaacs, Peter Sarnak and Kannan Soundararajan for helpful discussions.

\section{A CRITERION FOR VERIFYing ARTIN'S CONJECTURE}

Let $\rho: \operatorname{Gal}(K / \mathbb{Q}) \rightarrow \mathrm{GL}_{n}(\mathbb{C})$ be a Galois representation, as in the introduction. Brauer's theorem expresses the $L$-function $L(s, \rho)$ as a ratio $N(s) / D(s)$, where $N(s)$ and $D(s)$ are Artin $L$-functions associated to sums of monomial representations. If $f(s)$ is any holomorphic Artin $L$-function, we have a formula for the number $N_{f}\left(t_{1}, t_{2}\right)$ of zeros of $f$ between heights $t_{1}$ and $t_{2}$, from the argument principle:

$$
N_{f}\left(t_{1}, t_{2}\right)=\frac{1}{2 \pi i} \int_{C} \frac{f^{\prime}}{f}(s) d s,
$$

where $C$ is the rectangular contour with vertices at $2+i t_{1}, 2+i t_{2},-1+i t_{2},-1+i t_{1}$ and counter-clockwise orientation. We also have available in this case algorithms to compute $f$ and $f^{\prime}$ at an arbitrary point in the complex plane; see Section 5. Thus, in principle we could compute (2.1) exactly by numerical integration. Although (2.1) has the advantage of applying in great generality, to do so would be inefficient and difficult to implement rigorously. In the special case that the zeros of $f$ are simple, a much more efficient algorithm was given by Turing; see Section 4 .

No matter how we arrive at the numbers $N_{f}\left(t_{1}, t_{2}\right)$, there is always some uncertainty in the locations of the zeros of $f$. In (2.1) this is due to the fact that as $t_{i}$ approaches the ordinate of a zero, higher and higher precision is needed in order to compute $f^{\prime} / f$ accurately. This is in line with the expectation that the general zero is transcendental, meaning that one can never know it exactly.

For $L(s, \rho)$, we can recover the net number of zeros (i.e. zeros minus poles) between heights $t_{1}$ and $t_{2}$ as $N_{N}\left(t_{1}, t_{2}\right)-N_{D}\left(t_{1}, t_{2}\right)$. If Artin's conjecture is true then for every zero of $D(s)$ there is a zero of $N(s)$ at the same point. However, because of the uncertainty in the locations of the zeros of $N(s)$ and $D(s)$, from this computation alone we cannot rule out the possibility that $L(s, \rho)$ has a pole with a zero very close by in the neighborhood of a zero of $D(s)$. In other words, we can only observe the counts of net zeros in these small neighborhoods. 
Fortunately, there are some restrictions on potential poles. For instance, the Dedekind zeta function of the extension, $\zeta_{K}(s)$, factors into Artin $L$-functions:

$$
\zeta_{K}(s)=\prod_{\rho} L(s, \rho)^{\operatorname{dim} \rho},
$$

where the product is over all irreducible representations of $\operatorname{Gal}(K / \mathbb{Q})$. Since $\zeta_{K}(s)$ is holomorphic (except for a simple pole at $s=1$ ), we see that any pole of $L(s, \rho)$ in the critical strip must be located at the zero of another function. More generally, if $\sigma$ is any representation, we have

$$
L(s, \sigma)=\prod_{\rho} L(s, \rho)^{\langle\sigma, \rho\rangle},
$$

where $\langle\cdot, \cdot\rangle$ is the inner product on the space of characters, and by abuse of notation we write $\langle\sigma, \rho\rangle$ for $\langle\operatorname{Tr} \sigma, \operatorname{Tr} \rho\rangle$. When $\sigma$ is monomial, we again have $L(s, \sigma)$ holomorphic with the possible exception of a pole at $s=1$.

This information is described most concisely by use of the Heilbronn (virtual) character: For $s_{0} \in \mathbb{C} \backslash\{1\}$, define

$$
\theta_{s_{0}}=\sum_{\rho} \operatorname{ord}_{s=s_{0}} L(s, \rho) \cdot \operatorname{Tr} \rho
$$

where $\operatorname{ord}_{s=s_{0}} L(s, \rho):=\operatorname{Res}_{s=s_{0}} \frac{L^{\prime}}{L}(s, \rho)$. Thus,

$$
\operatorname{ord}_{s=s_{0}} L(s, \sigma)=\left\langle\theta_{s_{0}}, \sigma\right\rangle \geq 0 \text { for all monomial } \sigma .
$$

The study of Heilbronn characters leads to many useful results. For example, in FM89] it is shown that

$$
\sum_{\rho}\left(\operatorname{ord}_{s=s_{0}} L(s, \rho)\right)^{2} \leq\left(\operatorname{ord}_{s=s_{0}} \zeta_{K}(s)\right)^{2}
$$

In particular, the zeros and poles of each $L(s, \rho)$ are among the zeros of $\zeta_{K}(s)$.

The idea now is to combine (2.5) with observations of net zeros. If we look in a small enough neighborhood of a zero of $\zeta_{K}(s)$, we expect to find one net zero for a single $L(s, \rho)$ and no net zeros for the others. This is based on the assumption that the zeros of different irreducible Artin $L$-functions are distinct and simple. While such a statement is likely impossible to prove, we may use it as a working hypothesis to be tested at run time. This is analogous to assuming the simplicity of the zeros of $\zeta$ in order to check the Riemann hypothesis. (Note that if there were a multiple zero of $\zeta$, it is doubtful that one could distinguish it from a counterexample.)

In other words, if the working hypothesis is true, then our net zero observations correspond to the character $\operatorname{Tr} \rho$ for some $\rho$. Thus, we have $\operatorname{Tr} \rho=\theta_{s_{1}}+\ldots+\theta_{s_{n}}$, where $s_{1}, \ldots, s_{n}$ are the distinct zeros of $\zeta_{K}(s)$ in the neighborhood that we examine. We would like to conclude that there is just one such point, meaning that the actual zero counts agree 
with our observations. Since the Heilbronn characters satisfy (2.5), it is enough to show that

$$
\operatorname{Tr} \rho \neq \chi_{1}+\chi_{2} \text { for virtual characters } \chi_{i} \neq 0 \text { with }\left\langle\chi_{i}, \sigma\right\rangle \geq 0 \text { for all monomial } \sigma .
$$

The one notable exception to this philosophy is at the central point $\frac{1}{2}$, where there can be forced vanishing if $\rho$ is self-dual (an example of which is given in Arm72). In that case, we expect one zero for each self-dual $\rho$ with an odd functional equation, and no zeros for the rest. However, we can only determine the parity of the order of vanishing at $\frac{1}{2}$. This leads to the following replacement for condition (2.7) at $\frac{1}{2}$ :

$$
\sum_{\substack{\rho \text { self }- \text { dual } \\ \Lambda(1-s, \rho)=-\Lambda(s, \rho)}} \operatorname{Tr} \rho \neq \chi_{1}+2 \chi_{2} \quad \text { with } \chi_{i} \neq 0 \text { and }\left\langle\chi_{i}, \sigma\right\rangle \geq 0 \text { for all monomial } \sigma .
$$

When (2.7) is satisfied for all irreducible representations $\rho$, we may check the holomorphy of all $L(s, \rho)$ at any point at which the working hypothesis turns out to be true. We give a name to describe this situation:

Definition 2.1. A finite group $G$ is almost monomial if, for each irreducible representation $\rho$, if $\operatorname{Tr} \rho=\chi_{1}+\chi_{2}$ for virtual characters $\chi_{i}$ such that $\left\langle\chi_{i}, \sigma\right\rangle \geq 0$ for all monomial $\sigma$, then either $\chi_{1}=0$ or $\chi_{2}=0$.

The terminology is explained with aid of Figure 2.1. The plane represents the lattice of virtual characters, with the first quadrant being the monoid of characters, and the shaded cone the monoid generated by the monomial characters. We consider all virtual characters within 90 degrees of the cone, which in the figure is everything within the dashed lines. The group is almost monomial if this set is not much larger than the character monoid, in the precise sense that the irreducible representations, which are the coordinate axis vectors represented by thick arrows, remain indecomposable in this set. Equivalently, the monoid generated by the monomial characters should be close to the full character monoid. From the picture it is easy to see that any monomial group is almost monomial.

One could argue that we should include condition (2.8) in our definition as well. We prefer to keep it separate, taking the view that it is more important to be able to demonstrate holomorphy at a generic zero of the denominator. Indeed, we have already seen that the $L$-function of a 2-dimensional representation cannot have a finite number of poles, so we do not lose much generality by excluding a single point. It is plausible that such a result holds for higher dimensions as well. Moreover, condition (2.8) seems usually to be weaker than almost monomiality; cf. Proposition 2.3 below.

A potentially more serious issue is that $N(s)$ and $D(s)$ may have high order zeros at $\frac{1}{2}$, in which case Turing's method does not apply. This could be remedied by computing the contour integral (2.1) around $\frac{1}{2}$, but we would like to avoid doing so. Fortunately, if the order of vanishing at $\frac{1}{2}$ is at most 3 , we can still conclude that we have the correct count by sign changes alone; that is because for a self-dual representation, if we miss a zero away 


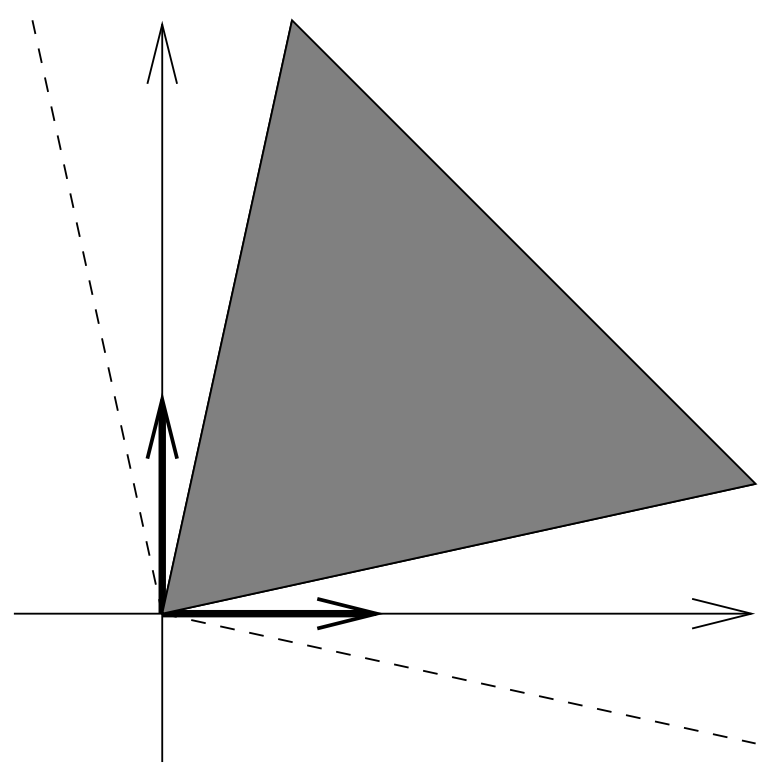

FIGURE 2.1. Lattice of virtual characters. The shaded cone is the monoid generated by monomial characters.

from $\frac{1}{2}$ then we must miss at least four such zeros. Fortunately again, in all cases that we consider, $N(s)$ has at most three irreducible factors with a potential zero at $\frac{1}{2}$.

Like monomiality, the notion of almost monomiality behaves well under some common group operations. In particular, we have the following.

Proposition 2.2. If $G$ is almost monomial then so are quotients of $G$ and products $G \times H$ for any monomial group $H$.

Proof. 1. Let $K$ be a normal subgroup of $G$ and $\tilde{\pi}$ an irreducible representation of $G / K$. Suppose that $\operatorname{Tr} \tilde{\pi}=\tilde{\chi}_{1}+\tilde{\chi}_{2}$, with $\left\langle\tilde{\chi}_{i}, \tilde{\sigma}\right\rangle \geq 0$ for all monomial $\tilde{\sigma}$. Let $\pi, \chi_{i}$ be the lifts of $\tilde{\pi}, \tilde{\chi}_{i}$ to $G$ obtained by composition with the natural projection. Then $\pi$ is irreducible and $\operatorname{Tr} \pi=\chi_{1}+\chi_{2}$. Further, if $\rho$ is an irreducible representation of $G$ then $\left\langle\chi_{i}, \rho\right\rangle=0$ unless $\rho$ factors through $G / K$. If that is the case, let $\tilde{\rho}$ denote the induced map on $G / K$.

Now, if $\sigma=\operatorname{Ind}_{H}^{G} \lambda$ is a monomial representation then, by Frobenius reciprocity, we have $\langle\sigma, \rho\rangle=\left\langle\operatorname{Res}_{H}^{G} \rho, \lambda\right\rangle$ for all $\rho$ factoring through $G / K$. If at least one of these is non-zero, i.e. $\lambda$ occurs in $\operatorname{Res}_{H}^{G} \rho$, then since $\rho$ factors through $G / K, \lambda$ must factor through $H / H \cap K \cong$ $H K / K$. Let $\tilde{\lambda}$ denote the induced map on $H K / K$. Then $\left\langle\operatorname{Res}_{H K / K}^{G / K} \tilde{\rho}, \tilde{\lambda}\right\rangle=\left\langle\operatorname{Res}_{H}^{G} \rho, \lambda\right\rangle$. Thus, $\tilde{\sigma}=\operatorname{Ind}_{H K / K}^{G / K} \tilde{\lambda}$ satisfies $\langle\sigma, \rho\rangle=\langle\tilde{\sigma}, \tilde{\rho}\rangle$. Therefore, $\left\langle\chi_{i}, \sigma\right\rangle=\left\langle\tilde{\chi}_{i}, \tilde{\sigma}\right\rangle \geq 0$. The conclusion follows by almost monomiality of $G$.

2. Let $\rho_{G}$ and $\rho_{H}$ be irreducible representations of $G$ and $H$, respectively, and suppose that $\operatorname{Tr} \rho_{G} \otimes \rho_{H}=\left(\operatorname{Tr} \rho_{G}\right)\left(\operatorname{Tr} \rho_{H}\right)=\chi_{1}+\chi_{2}$ with $\left\langle\chi_{i}, \sigma\right\rangle_{G \times H} \geq 0$ for all monomial $\sigma$. Taking the inner product over $H$ with $\rho_{H}$, we get $\operatorname{Tr} \rho_{G}=\left\langle\chi_{1}, \rho_{H}\right\rangle_{H}+\left\langle\chi_{2}, \rho_{H}\right\rangle_{H}$. 
Next, if $\sigma_{G}$ is any monomial representation of $G$, we have $\left\langle\left\langle\chi_{i}, \rho_{H}\right\rangle_{H}, \sigma_{G}\right\rangle_{G}=\left\langle\chi_{i}, \sigma_{G} \otimes\right.$ $\left.\rho_{H}\right\rangle_{G \times H} \geq 0$, since $\sigma_{G} \otimes \rho_{H}$ is monomial. Thus, since $G$ is almost monomial, we have $\left\langle\chi_{i}, \rho_{H}\right\rangle=0$ for some $i$.

Similarly, if $\rho_{H}^{\prime}$ is any other irreducible representation of $H$, we find $0=\left\langle\chi_{1}, \rho_{H}^{\prime}\right\rangle_{H}+$ $\left\langle\chi_{2}, \rho_{H}^{\prime}\right\rangle_{H}$. Thus, $\left\langle\chi_{1}, \rho_{H}^{\prime}\right\rangle=\left\langle\chi_{2}, \rho_{H}^{\prime}\right\rangle=0$. Therefore $\chi_{i}=0$ for some $i$.

The next proposition shows that the class of almost monomial groups is strictly larger than that of monomial groups.

Proposition 2.3. The groups $\mathrm{SL}_{2}\left(\mathbb{F}_{3}\right), A_{5}$ and $S_{5}$ are almost monomial and satisfy (2.8).

Proof. These are shown with the aid of the computer algebra system GAP [GAP05]. We illustrate the general procedure for checking almost monomiality for a given group with the example $A_{5}$. Note first that $A_{5}$ has five irreducible representations, of dimensions 1 , 3, 3, 4 and 5. We use GAP to determine all monomial representations. In this case they are non-negative linear combinations of the vectors $(1,0,0,0,0),(0,0,0,0,1),(1,0,0,1,0)$, $(0,1,1,0,0),(0,1,0,1,1),(0,0,1,1,1)$, and $(0,1,1,1,0)$, where the components indicate the multiplicities of the irreducible representations. We label the monomial representations associated to these vectors $\sigma_{1}, \ldots, \sigma_{7}$. The first five form a $\mathbb{Z}$-basis for the lattice of virtual characters, i.e. any virtual character $\chi$ may be written uniquely as an integral linear combination $\chi=\sum_{i=1}^{5} x_{i} \operatorname{Tr} \sigma_{i}$.

Now, almost monomiality is equivalent to the assertion that for each irreducible representation $\rho$, whenever $0 \leq\langle\chi, \sigma\rangle \leq\langle\rho, \sigma\rangle$ for all monomial $\sigma$, we have either $\chi=0$ or $\chi=\operatorname{Tr} \rho$. Using our integral basis, we investigate the solutions to

$$
0 \leq \sum_{i=1}^{5} x_{i}\left\langle\sigma_{i}, \sigma_{j}\right\rangle \leq\left\langle\rho, \sigma_{j}\right\rangle
$$

for $j=1, \ldots, 7$. Restricting to $j=1, \ldots, 5$, we get an invertible system, i.e. the matrix $A=$ $\left(\left\langle\sigma_{i}, \sigma_{j}\right\rangle\right)_{1 \leq i, j \leq 5}$ lies in $\mathrm{SL}_{5}(\mathbb{Z})$. We consider the vectors $\mathbf{x}=A^{-1} \mathbf{y}$ for all $\mathbf{y}=\left(y_{1}, \ldots, y_{5}\right)$ satisfying $0 \leq y_{j} \leq\left\langle\rho, \sigma_{j}\right\rangle$. By construction, these satisfy (2.9) for $j=1, \ldots, 5$. We check that the only $\mathrm{x}$ satisfying (2.9) for $j=6,7$ are 0 and $A^{-1}\left(\left\langle\rho, \sigma_{j}\right\rangle\right)$, corresponding to $\chi=0$ and $\chi=\operatorname{Tr} \rho$, respectively.

Similarly, for (2.8) we try all possible combinations of $\rho$ having odd functional equation. We may exclude those whose $L$-functions may be expressed in terms of Dedekind zeta functions, for which the root number is always 1 . For $A_{5}$, the only non-trivial possibility is that the two 3-dimensional representations have odd functional equation.

With the evidence provided by Propositions 2.2 and 2.3, one might hope that all groups are almost monomial. That is not the case, as the counterexamples $\mathrm{GL}_{2}\left(\mathbb{F}_{3}\right)$ and $\mathrm{SL}_{2}\left(\mathbb{F}_{5}\right)$ show. $\mathrm{SL}_{2}\left(\mathbb{F}_{5}\right)$ has irreducible representations of dimensions $1,2,2,3,3,4,4,5$ and 6 , and it is the smallest group supporting an icosahedral representation (since $A_{5}$ has no 2 dimensional representations), meaning that our criterion unfortunately does not apply to 
checking the icosahedral case. In fact, one knows Artin's conjecture for all induced representations of this group; while they are not all monomial, the only exceptions come from a pair of tetrahedral representations, for which we have the Langlands-Tunnell theorem. Even with this added information, we cannot rule out the possibility of a simple pole with undetectably small residue at a zero of the $L$-function of the 6-dimensional representation. More precisely, we find with GAP that the induced representations are spanned by the twelve vectors

$$
\begin{array}{lll}
(1,0,0,0,0,0,0,0,0) & (0,0,0,0,0,0,0,1,0) & (1,0,0,0,0,1,0,0,0) \\
(0,0,0,1,1,0,0,0,0) & (0,0,0,1,0,1,0,1,0) & (0,0,0,0,1,1,0,1,0) \\
(0,0,0,1,1,1,0,0,0) & (0,0,0,0,0,0,0,0,1) & (0,0,0,0,0,0,1,0,1) \\
(0,1,0,0,0,0,1,0,1) & (0,0,1,0,0,0,1,0,1) & (0,1,1,0,0,0,0,0,1) .
\end{array}
$$

The first seven of these are the monomial representations lifted from $\mathrm{SL}_{2}\left(\mathbb{F}_{5}\right) /\{ \pm I\} \cong A_{5}$, while the others give "new" information. One easily checks that for $\rho$ the 6 -dimensional representation, (2.7) fails with $\chi_{1}$ corresponding to any of the vectors $(0,-1,0,0,0,0,0,0,1)$, $(0,0,-1,0,0,0,0,0,1)$ and $(0,0,0,0,0,0,-1,0,1)$, i.e. the representations of dimension 2 and one of dimension 4 can hide a pole at a zero of $L(s, \rho)$. This shows in a strong sense that information from induced representations is in general insufficient to show Artin's conjecture.

However, all is not lost concerning icosahedral representations. For a given icosahedral $\rho$, the adjoint square $\operatorname{Ad}(\rho)$ is a 3-dimensional representation with image isomorphic to $A_{5}$. A result of Flicker [Fli94] implies that modularity of $\rho$ is equivalent to that of $\operatorname{Ad}(\rho)$. (In fact, modularity of all representations of the underlying group follows from that of $\operatorname{Ad}(\rho)$ and its Galois conjugate, by known cases of functoriality; see Wan03.) Combining this fact with the GL(3) converse theorem, one could give a converse theorem for GL(2) using analytic properties of $L(s, \operatorname{Ad}(\rho) \otimes \chi)$ for Dirichlet characters $\chi$. Weissman, in his undergraduate thesis Wei99, used this idea to give indirect evidence for the modularity of an even icosahedral representation. By Propositions 2.2 and 2.3 we see that in principle we may directly verify the holomorphy of these $L$-functions up to finite height. Moreover, an "effective" version of the GL(3) converse theorem (requiring, say, meromorphy of all twists and holomorphy of a finite number in a bounded region) would suffice to give an algorithm for verifying the conjecture in the icosahedral case.

Unfortunately, there is the more practical problem that totally real $A_{5}$ fields (those that give rise to even icosahedral representations) are very rare; the smallest known discriminant is far too large to test with current computers. Thus, for the $A_{5}$ examples that we consider in Section [6] the Artin conjecture is already known. To test our criterion, we consider instead some examples of $S_{5}$ extensions, which exist in much greater abundance.

Finally, we note that in the course of verifying Artin's conjecture, the information that we collect implies that the zeros of each $L(s, \rho)$ are simple and lie on the line $\Re(s)=\frac{1}{2}$. Thus, in the process we also verify the Riemann hypothesis for $\zeta_{K}(s)$. Interestingly, we do not need to establish the holomorphy of all $L(s, \rho)$ in order to do this; it is enough, 
for example, that they have at most simple poles. More precisely, in order to check the Riemann hypothesis around a generic zero of $\zeta_{K}(s)$ we need to have

$$
\operatorname{Tr} \rho \neq \chi_{1}+2 \chi_{2} \text { for virtual characters } \chi_{i} \neq 0 \text { with }\left\langle\chi_{i}, \sigma\right\rangle \geq 0 \text { for all monomial } \sigma,
$$

which is a weaker condition than almost monomiality. In particular, we may still check the Riemann hypothesis for $\mathrm{SL}_{2}\left(\mathbb{F}_{5}\right)$ extensions.

\section{LOCATING ZEROS VIA THE EXPLiCit FORMULA}

Let notation be as in the introduction, and define numbers $c_{n}$ by $-\frac{L^{\prime}}{L}(s)=\sum_{n=1}^{\infty} c_{n} n^{-s}$, i.e. $c_{n}=(\log p) \sum_{j=1}^{r} \alpha_{p, j}^{k}$ for $n=p^{k}$ a prime power, and $c_{n}=0$ otherwise. Further, we enumerate the zeros of $\Lambda(s)$ as $\rho_{n}=\frac{1}{2}+i \gamma_{n}$ for $n \in \mathbb{Z}$, repeated with multiplicity. Weil's explicit formula relates the sequences $\left\{c_{n}\right\}$ and $\left\{\gamma_{n}\right\}$. Precisely, let $g \in C_{c}^{1}(\mathbb{R})$ be a differentiable function of compact support such that its Fourier transform $h(t):=$ $\int_{-\infty}^{\infty} g(x) e^{i x t} d x$ is real for $t \in \mathbb{R}$. Then

$$
\begin{aligned}
& \sum_{n \in \mathbb{Z}} h\left(\gamma_{n}\right)-2 \Re \sum_{k=1}^{m} h\left(-i\left(\frac{1}{2}+\lambda_{k}\right)\right) \\
& \quad=g(0) \log N+2 \Re\left[\sum_{j=1}^{r} \frac{1}{2 \pi} \int_{-\infty}^{\infty} \frac{\Gamma_{\mathbb{R}}^{\prime}}{\Gamma_{\mathbb{R}}}\left(\frac{1}{2}+\mu_{j}+i t\right) h(t) d t-\sum_{n=1}^{\infty} \frac{c_{n}}{\sqrt{n}} g(\log n)\right],
\end{aligned}
$$

This follows from the Cauchy integral formula and the functional equation; see RS96. Note that all terms of the formula may be put in terms of $g$; in particular,

$$
\frac{1}{2 \pi} \int_{-\infty}^{\infty} \frac{\Gamma_{\mathbb{R}}^{\prime}}{\Gamma_{\mathbb{R}}}\left(\frac{1}{2}+\mu+i t\right) h(t) d t=\frac{1}{2} \int_{0}^{\infty} \log \left(\pi e^{\gamma}\left(e^{2 x}-1\right)\right) d\left(g(x) e^{-(1 / 2+\mu) x}\right) .
$$

This form is convenient for computation, since $g$ has compact support.

The important thing to note is that given a list of the $c_{n}$ for $n \leq e^{X}$, the explicit formula gives a method for evaluating $\sum_{n} h\left(\gamma_{n}\right)$ for essentially any function $h$ whose Fourier transform is supported in $[-X, X]$. When $X$ is large, we may choose $h$ to be narrowly concentrated around any particular point, and thus resolve features of the spectrum in places where the density of zeros is not too large compared to $X$; a variant of this technique, with explicit test functions (not of compact support), was worked out by Omar Oma01. to estimate the lowest zero of some Dedekind zeta functions. For a fixed support $[-X, X]$, there is a canonical way of choosing a "best" test function, by a method developed for the Selberg trace formula in BS05]. In order to use the method, which depends crucially on a positivity argument, it is necessary to assume the Riemann hypothesis for our given $L$-function. With that caveat, we recall briefly the construction from [BS05].

For $t_{0} \in \mathbb{R}$, let $\mathcal{C}\left(X, t_{0}\right)$ be the class of functions $h$ as above, with the corresponding $g$ supported in $[-X, X]$, and the additional restrictions $h(t) \geq 0$ for $t \in \mathbb{R}$ and $h\left(t_{0}\right)=1$. 
Define

$$
F_{X}\left(t_{0}\right):=\inf _{h \in \mathcal{C}\left(X, t_{0}\right)} \sum_{n \in \mathbb{Z}} h\left(\gamma_{n}\right)
$$

Then as $X \rightarrow \infty, F_{X}$ tends pointwise to the characteristic function of the zeros. Moreover, if $F_{X}\left(t_{0}\right)<1$ for any value of $X$ then $t_{0}$ cannot be the ordinate of a zero. Thus, by evaluating $F_{X}$ we can find provable intervals in which the zeros must lie.

Although the definition of $F_{X}$ is abstract, it is easy to construct concrete families of functions that closely approximate any desired function. For instance, let $M$ be a large integer, $\delta=X / 2 M$ and set

$$
h(t)=\left(\frac{\sin \delta t / 2}{\delta t / 2}\right)^{4}\left(a_{0}+\sum_{n=1}^{M-1}\left(a_{n} \cos \delta n t+b_{n} \sin \delta n t\right)\right)^{2},
$$

for arbitrary real numbers $a_{n}, b_{n}$. (For self-dual $L$-functions, we restrict to even test functions, i.e. all $b_{n}=0$, and divide the final formula by 2.) On the other side of the Fourier transform, this corresponds to taking $g=f * f$, where $f$ linearly interpolates arbitrary values at multiples of $\delta$.

The sum over zeros in (3.3) is then a positive definite quadratic form in the numbers $a_{n}$ and $b_{n}$. To compute the matrix of the form essentially involves computing the explicit formula for functions $g$ that are translates of a fixed function of small compact support. That requires almost no extra work, since we may compute the formula for all localized test functions simultaneously. Once the matrix is known, the infemum in (3.3) over this restricted class of test functions is easily found as the minimum of the quadratic form subject to the linear constraint $h\left(t_{0}\right)=1$. This involves inverting the matrix, after which the minimum may be found quickly for many different values of $t_{0}$.

For an $L$-function of degree $r$ and conductor $N$, the density of zeros at height $T$ is roughly $\frac{1}{2 \pi} \log N\left(\frac{T}{2 \pi}\right)^{r}$. Therefore, in order to resolve features around height $T$, the uncertainty principle says we should know the numbers $c_{n}$ for $n$ up to about $N\left(\frac{T}{2 \pi}\right)^{r}$. In the selfdual case, the extra division by 2 replaces this by its square root; thus, the complexity is on par with that of the approximate functional equation or the algorithm of Section 5. although it is much more sensitive to the local spacing of zeros. (Heuristic arguments based on experiments and random matrix theory Odl87] indicate that the minimum gap between zeros can be arbitrarily small relative to the mean value; although such small gaps are expected to be very rare, we could in principle need many more coefficients than for the "typical" zero at height $T$.) In practice, the explicit formula is clean and easy to implement since there are no error terms to estimate with functions of compact support. It is particularly well-suited to finding low zeros or to situations where the numbers $c_{n}$ may be computed quickly, as is the case for Artin $L$-functions; cf. Section 6.1.

As mentioned above, the minimization procedure requires assuming the Riemann hypothesis. If one is willing to do so, the method may be made completely rigorous, and may even be used in place of Turing's method for verifying Artin's conjecture. However, it is 
more natural to use it as a quick check in order to fine tune and validate the subsequent rigorous methods. In fact, it is helpful to assume Artin's conjecture and apply the method to the irreducible Artin $L$-functions directly. That thins out the spectrum, making it easier to isolate individual zeros. We have carried out this procedure for a few examples in Section 6.2

\section{TURING'S METHOD}

Turing's method for verifying the Riemann hypothesis is described well in his paper Tur53, although there are some errors in the details that were later corrected by Lehman Leh70. The method has subsequently been extended to Dirichlet $L$-functions by Rumely Rum93 and Dedekind zeta functions by Tollis $[\text { Tol97 }]^{2}$. Our contribution is to work out the details necessary to apply it to an arbitrary $L$-function with simple zeros.

Our argument essentially follows that of Turing. We begin by setting some notation to be used only in this section. For $t$ not the ordinate of a zero or pole of $\Lambda$, let

$$
S(t):=\frac{1}{\pi} \Im \int_{\infty}^{1 / 2} \frac{L^{\prime}}{L}(\sigma+i t) d \sigma .
$$

By convention, we make $S(t)$ upper semi-continuous, i.e. when $t$ is the ordinate of zero or pole, we define $S(t)=\lim _{\varepsilon \rightarrow 0^{+}} S(t+\varepsilon)$.

Next, for $t_{1}<t_{2}$ let $N\left(t_{1}, t_{2}\right)$ denote the net number of zeros with imaginary part in $\left(t_{1}, t_{2}\right]$, counting multiplicity. When neither $t_{1}$ nor $t_{2}$ is the ordinate of a zero or pole, we may calculate $N\left(t_{1}, t_{2}\right)$ using the argument principle, as in (2.1). Let $C$ be the rectangle with corners at $2+i t_{1}, 2+i t_{2},-1+i t_{2},-1+i t_{1}$, with counter-clockwise orientation, $H$ the half plane $\left\{s \in \mathbb{C}: \Re(s) \geq \frac{1}{2}\right\}$, and $H^{c}$ its complement. Note that by the functional equation, we have $\overline{\frac{\Lambda^{\prime}}{\Lambda}(s)}=-\frac{\Lambda^{\prime}}{\Lambda}(1-\bar{s})$. Hence,

$$
\begin{aligned}
N\left(t_{1}, t_{2}\right) & =\frac{1}{2 \pi} \Im \int_{C} \frac{\Lambda^{\prime}}{\Lambda}(s) d s=\frac{1}{2 \pi} \Im\left(\int_{C \cap H} \frac{\Lambda^{\prime}}{\Lambda}(s) d s-\overline{\int_{C \cap H^{c}}} \frac{\Lambda^{\prime}}{\Lambda}(s) d s\right) \\
& =\frac{1}{2 \pi} \Im\left(\int_{C \cap H} \frac{\Lambda^{\prime}}{\Lambda}(s) d s+\int_{C \cap H^{c}} \frac{\Lambda^{\prime}}{\Lambda}(1-\bar{s}) d \bar{s}\right) \\
& =\frac{1}{\pi} \Im \int_{C \cap H} \frac{\Lambda^{\prime}}{\Lambda}(s) d s=\frac{1}{\pi} \Im \int_{C \cap H} \frac{\gamma^{\prime}}{\gamma}(s) d s+\frac{1}{\pi} \Im \int_{C \cap H} \frac{L^{\prime}}{L}(s) d s .
\end{aligned}
$$

Now for the integral of $L^{\prime} / L$ we move the right edge of the contour out to $\infty$, where the integrand vanishes. We thus obtain

$$
N\left(t_{1}, t_{2}\right)=\left.\frac{1}{\pi} \Im \log \gamma(s)\right|_{\frac{1}{2}+i t_{1}} ^{\frac{1}{2}+i t_{2}}+S\left(t_{2}\right)-S\left(t_{1}\right) .
$$

\footnotetext{
${ }^{2}$ Tollis applied his method to cubic and quartic fields. In these cases, there is a slight advantage in passing to the normal closure and separating into irreducible Artin $L$-functions, as we have done for the $A_{5}$ cases in Section 6
} 
We select a particular branch of $\log \gamma(s)$ by using the principal branch of $\log \Gamma$. With this choice, set

$$
\Phi(t):=\frac{1}{\pi}\left[\arg \epsilon+\frac{\log N}{2} t-\frac{\log \pi}{2}\left(r t+\Im \sum_{j=1}^{r} \mu_{j}\right)+\Im \sum_{j=1}^{r} \log \Gamma\left(\frac{1 / 2+i t+\mu_{j}}{2}\right)\right]
$$

and

$$
N(t):=\Phi(t)+S(t) .
$$

Then $N\left(t_{1}, t_{2}\right)=N\left(t_{2}\right)-N\left(t_{1}\right)$. Note that if $L$ is self-dual and vanishes to order $\leq 1$ at $\frac{1}{2}$ then $N(t)=N(0, t)$. In the general case, although we still have $N(t) \in \mathbb{Z}$, there is no standard reference point, so only changes in $N(t)$ are meaningful. (Put another way, the branch of $\log \gamma$ chosen in (4.4) is non-canonical.) For large $t, \Phi(t)$ may be evaluated quickly by an effective version of Stirling's formula:

$$
\Im \log \Gamma(z)=\Im\left[\left(z-\frac{1}{2}\right) \log \frac{z}{e}\right]+\Theta\left(\frac{1}{8|\Im(z)|}\right) \quad \text { for } z \in \mathbb{C} \backslash \mathbb{R},
$$

where the notation $f=\Theta(g)$ means $|f| \leq g$.

Turing's method is as follows. Recall that $\Lambda\left(\frac{1}{2}+i t\right)$ is real valued. Thus, if we have an accurate procedure to compute $\Lambda(s)$ then we may locate all simple zeros on the line $\Re(s)=\frac{1}{2}$ by observing its sign changes. If it turns out that all of the zeros between ordinates $t_{1}$ and $t_{2}$ are simple and on the line, then we can deduce the Riemann hypothesis in that interval by computing $N\left(t_{1}, t_{2}\right)$ (minus the contribution from any poles between $t_{1}$ and $\left.t_{2}\right)$ and finding the same number of sign changes over the interval.

To compute $N\left(t_{1}, t_{2}\right)$, we could evaluate (4.2) numerically. However, this would require many evaluations of $\Lambda(s)$ and would be difficult to carry out rigorously. Fortunately, Turing devised a simpler method, based on the fact (first due to Littlewood for $\zeta(s)$ ) that $S(t)$ has mean value 0 . Thus, the graph of $N\left(t_{0}, t\right)-\Phi(t)$ for any fixed $t_{0}$ oscillates around a constant value; if we were to plot the same function using the measured number of zeros in $\left(t_{0}, t\right]$, then any zeros that we had missed would be obvious as jumps in the graph.

This can be made rigorous as follows. Let $t_{0}$ be a large number that is not the ordinate of a zero or pole, and assume that between ordinates $t_{0}-h$ and $t_{0}+h$ (for some $h>0$ ), we have located several zeros of $\Lambda(s)$, i.e. we have found small intervals $\left(a_{n}, b_{n}\right)$ such that $\Lambda\left(\frac{1}{2}+i a_{n}\right)$ and $\Lambda\left(\frac{1}{2}+i b_{n}\right)$ have opposite sign. Let $N_{\text {left }}\left(t_{0}, t\right)$ (resp. $\left.N_{\text {right }}\left(t_{0}, t\right)\right)$ be the step function which is upper semi-continuous, increases by 1 at each $a_{n}$ (resp. $b_{n}$ ) and vanishes at $t=t_{0}$. We then have

$$
N(t) \leq N\left(t_{0}\right)+N_{\text {left }}\left(t_{0}, t\right) \text { for } t \leq t_{0} \quad \text { and } \quad N(t) \geq N\left(t_{0}\right)+N_{\text {right }}\left(t_{0}, t\right) \text { for } t \geq t_{0} .
$$

From these, we can deduce upper and lower bounds for $N\left(t_{0}\right)$; integrating (4.7), we get

$$
N\left(t_{0}\right) h+\int_{t_{0}}^{t_{0}+h} N_{\text {right }}\left(t_{0}, t\right) d t \leq \int_{t_{0}}^{t_{0}+h} N(t) d t=\int_{t_{0}}^{t_{0}+h} \Phi(t) d t+\int_{t_{0}}^{t_{0}+h} S(t) d t
$$


and

$$
N\left(t_{0}\right) h+\int_{t_{0}-h}^{t_{0}} N_{\text {left }}\left(t_{0}, t\right) d t \geq \int_{t_{0}-h}^{t_{0}} N(t) d t=\int_{t_{0}-h}^{t_{0}} \Phi(t) d t+\int_{t_{0}-h}^{t_{0}} S(t) d t .
$$

If we have in fact located all zeros in the interval $\left(t_{0}-h, t_{0}+h\right)$ with some amount of precision (as measured by the size of the intervals $\left(a_{n}, b_{n}\right)$ ), then we can expect these bounds to be close to the truth. Moreover, if we have effective upper and lower bounds for the integral of $S(t)$, then for $h$ large enough, (4.8) and (4.9) will bound a single integer, i.e. we can unambiguously determine $N\left(t_{0}\right)$. Doing this for two different values $t_{0}=t_{1}, t_{2}$, we obtain $N\left(t_{1}, t_{2}\right)$.

One nice feature of Turing's method is that precise knowledge of the zeros is only required in the short intervals around $t_{1}$ and $t_{2}$, and even there one can make a trade-off between the precision of the zeros and the length $h$ of the interval. For the bulk of the zeros between $t_{1}$ and $t_{2}$ it suffices to observe the sign changes.

The remainder of this section is devoted to bounding $\int S(t) d t$, cf. Theorem 4.5 below. Our starting point is the following formula, obtained by Littlewood's box principle (see Tit86, $\S 9.9])$ :

$$
\pi \int_{t_{1}}^{t_{2}} S(t) d t=\int_{1 / 2}^{\infty} \log \left|L\left(\sigma+i t_{2}\right)\right| d \sigma-\int_{1 / 2}^{\infty} \log \left|L\left(\sigma+i t_{1}\right)\right| d \sigma .
$$

Lemma 4.1. Let notation be as above, and set $B:=\sup _{\Re(s)=3 / 2}|L(s)|^{2}$. Then, for $s$ in the strip $\left\{s \in \mathbb{C}:-\frac{1}{2} \leq \Re(s) \leq \frac{3}{2}\right\}$,

$$
|L(s)|^{2} \leq B|\chi(s) Q(s)| \frac{P(s+1)^{2} P(s-2)}{P(s)^{2} P(s-1)} \mid .
$$

Remark. The power of $|Q(s)|$ in the above is not optimal; for $\Re(s)=\frac{1}{2}$, the "convexity bound" says that we can put instead $|Q(s)|^{1 / 2+\varepsilon}$, with a constant depending on $\varepsilon$ (see IS00 ), while the Lindelöf hypothesis would have $|Q(s)|^{\varepsilon}$. Our present choice permits us to avoid Stirling's formula in the proof, and thus obtain a clean bound that is uniform in all parameters.

Proof. We consider first the case when $L(s)$ is entire. Set

$$
F(s):=L(s) \bar{L}(1-s)=\chi(s)^{-1} L(s)^{2} .
$$

Plugging in the definition of $\chi(s)$,

$$
|F(\sigma+i t)|=|L(\sigma+i t)|^{2}\left|\frac{\gamma(\sigma+i t)}{\bar{\gamma}(1-\sigma-i t)}\right|=|L(\sigma+i t)|^{2}\left|\frac{\gamma(\sigma+i t)}{\gamma(1-\sigma+i t)}\right| .
$$

Note that when $\sigma=\frac{1}{2}+$ a positive integer, the ratio of $\gamma$ factors reduces to a polynomial; in particular,

$$
|F(3 / 2+i t)|=|L(3 / 2+i t)|^{2}|Q(-1 / 2+i t)| \leq B|Q(3 / 2+i t)| .
$$


The inequality holds since $\Re\left(\mu_{j}\right) \geq-\frac{1}{2}$ for all $j$. Next, from the functional equation we have $F(s)=\bar{F}(1-s)$, so that

$$
|F(\sigma+i t)|=|F(1-\sigma+i t)| .
$$

Hence, by (4.14),

$$
|F(-1 / 2+i t)| \leq B|Q(-1 / 2+i t)|
$$

Thus, the function $F(s) / Q(s)$ is bounded by $B$ on the lines $\Re(s)=-\frac{1}{2}$ and $\Re(s)=\frac{3}{2}$. Note that although $Q(s)$ has zeros, $F(s)$ has trivial zeros at the same points; in fact

$$
\frac{F(s)}{Q(s)}=\frac{\Lambda(s) \bar{L}(1-s)}{\gamma(s) Q(s)}=\frac{\Lambda(s) \bar{L}(1-s)}{\gamma(s+2)} .
$$

Since $F$ has finite order, it follows from the Phragmen-Lindelöf theorem that $|F(s)| \leq$ $B|Q(s)|$ for all $s$ in the strip.

If $L(s)$ has poles then the above argument breaks down since $F(s) / Q(s)$ is not holomorphic in the strip. In fact, for each $k$ we get three poles, one at $1+\lambda_{k}$ and two at $\lambda_{k}$, as (4.17) shows. To compensate for this, we consider $F(s) P(s)^{2} P(s-1)$ in the above, in place of $F(s)$. One checks that $\left|s^{2}(s-1)\right| \leq\left|(s+1)^{2}(s-2)\right|$ on the lines $\Re(s)=-1 / 2$ and $\Re(s)=3 / 2$, so that

$$
\left|F(s) P(s)^{2} P(s-1)\right| \leq B\left|Q(s) P(s)^{2} P(s-1)\right| \leq B\left|Q(s) P(s+1)^{2} P(s-2)\right| .
$$

Further, the ratio $\frac{F(s) P(s)^{2} P(s-1)}{Q(s) P(s+1)^{2} P(s-2)}$ is holomorphic in the strip, so we may proceed as above. The lemma follows.

Lemma 4.2. Suppose that

$$
\left(t+\Im\left(\mu_{j}\right)\right)^{2} \geq\left(5 / 2+\Re\left(\mu_{j}\right)\right)^{2}+X^{2} \quad \text { for some } X>5 \text { and all } j=1, \ldots, r \text {. }
$$

Then

1. For $\sigma \in[1 / 2,5 / 2]$,

$$
-r\left(\frac{1}{2 \sqrt{2} X}+\frac{4 / \pi^{2}+1 / 4}{X^{2}}\right) \leq \Re \frac{\gamma^{\prime}}{\gamma}(\sigma+i t)-\frac{1}{2} \log \left|Q\left(\frac{3}{2}+i t\right)\right| \leq \frac{4 r}{\pi^{2} X^{2}} .
$$

2. For $\sigma \in[-1 / 2,3 / 2]$,

$$
\Re \frac{Q^{\prime}}{Q}(\sigma+i t) \leq \frac{r}{\sqrt{2} X} .
$$

3. For all $\sigma$,

$$
\Re \frac{P^{\prime}}{P}(\sigma+i t) \leq \frac{\max (\sigma m, 0)}{X^{2}} .
$$


Proof. 1. We have

$$
\frac{\gamma^{\prime}}{\gamma}(\sigma+i t)=\frac{1}{2} \log \frac{N}{\pi^{r}}+\frac{1}{2} \sum_{j=1}^{r} \frac{\Gamma^{\prime}}{\Gamma}\left(\frac{\sigma+i t+\mu_{j}}{2}\right) .
$$

We apply the Stirling-type estimate [Leh70]

$$
\frac{\Gamma^{\prime}}{\Gamma}(z)=\log z-\frac{1}{2 z}+\Theta\left(\frac{2 / \pi^{2}}{\left|\Im(z)^{2}-\Re(z)^{2}\right|}\right) \quad \text { for } \Re(z) \geq 0 .
$$

This yields

$$
\begin{aligned}
& \Re \frac{\gamma^{\prime}}{\gamma}(\sigma+i t)-\frac{1}{2} \log \left|Q\left(\frac{3}{2}+i t\right)\right| \\
& =-\frac{1}{2} \sum_{j=1}^{r}\left[\log \left|\frac{3 / 2+i t+\mu_{j}}{\sigma+i t+\mu_{j}}\right|+\Re \frac{1}{\sigma+i t+\mu_{j}}+\Theta\left(\frac{8 / \pi^{2}}{\left|\left(t+\Im\left(\mu_{j}\right)\right)^{2}-\left(\sigma+\Re\left(\mu_{j}\right)\right)^{2}\right|}\right)\right] .
\end{aligned}
$$

For the lower bound in (4.20) we need an upper bound for the expression in brackets. By hypothesis, the $\Theta$ term is bounded by $\frac{8}{\pi^{2} X^{2}}$. For the others, put $\sigma+i t+\mu_{j}=x+i y$, $\beta=3 / 2-\sigma$, so that $|\beta| \leq 1$ and $x$ and $y$ are constrained by $y^{2} \geq x^{2}+X^{2}, x \geq 0$. Then, using the inequality $\log (1+u) \leq u$, we have

$$
\begin{aligned}
\log \left|\frac{x+\beta+i y}{x+i y}\right|+\Re \frac{1}{x+i y} & =\frac{1}{2} \log \left(1+\frac{2 \beta x+\beta^{2}}{x^{2}+y^{2}}\right)+\frac{x}{x^{2}+y^{2}} \\
& \leq \frac{(\beta+1) x+\beta^{2} / 2}{x^{2}+y^{2}} \leq \frac{2 x+1 / 2}{2 x^{2}+X^{2}} \leq \frac{1}{\sqrt{2} X}+\frac{1}{2 X^{2}} .
\end{aligned}
$$

There are $r$ such terms, and the lower bound follows after multiplying by $-\frac{1}{2}$.

The upper bound is similar, but uses the second order inequality $\log (1+u) \geq u-u^{2} / 2$. We omit the details.

2. Similarly,

$$
\Re \frac{Q^{\prime}}{Q}(\sigma+i t)=\sum_{j=1}^{r} \Re \frac{1}{\sigma+\mu_{j}+i t} \leq \sum_{j=1}^{r} \frac{\sigma+\Re\left(\mu_{j}\right)}{2\left(\sigma+\Re\left(\mu_{j}\right)\right)^{2}+X^{2}} \leq \frac{r}{\sqrt{2} X} .
$$

3.

$$
\Re \frac{P^{\prime}}{P}(\sigma+i t)=\sum_{k=1}^{m} \Re \frac{1}{\sigma+i t-\lambda_{k}} \leq \sum_{k=1}^{m} \frac{\max (\sigma, 0)}{\sigma^{2}+X^{2}} \leq \frac{\max (\sigma m, 0)}{X^{2}} .
$$

(Note that this bound is of faster decay than estimates 1 and 2. That is because we have control over the real parts of the poles, while nothing prevents $\Re\left(\mu_{j}\right)$ from being of comparable size to $X$. One could also obtain an $O\left(X^{-2}\right)$ bound in 1 and 2 , with a constant depending on the $\mu_{j}$.) 
Lemma 4.3. Let $w$ be a complex number with $|\Re(w)| \leq \frac{1}{2}$. Then

$$
\int_{0}^{1} \log \left|\frac{(x+1+w)(x+1-\bar{w})}{(x+w)(x-\bar{w})}\right| d x \leq(\log 4) \Re\left(\frac{1}{1+w}+\frac{1}{1-\bar{w}}\right) .
$$

Proof (sketch). Note first that equality is attained at $w=0$. Using the principal branch of the logarithm, set

$$
f(w)=\int_{0}^{1} \log \left(\frac{(x+1+w)(x+1-w)}{(x+w)(x-w)}\right) d x \quad \text { and } \quad g(w)=\frac{1}{1+w}+\frac{1}{1-w} .
$$

These define analytic functions on $\mathbb{C} \backslash \mathbb{R}$, with real parts extending continuously to $\mathbb{R}$. Further, 4.29) is equivalent to the assertion

$$
\Re(f(w)-(\log 4) g(w)) \leq 0 \quad \text { for }|\Re(w)| \leq \frac{1}{2} .
$$

Note that $f(w)$ and $g(w)$ are each asymptotic to $-2 / w^{2}$ as $|w| \rightarrow \infty$. Thus, (4.31) holds for $\Im(w)$ sufficiently large; we check that in fact $|\Im(w)| \geq 2$ is enough. By symmetry and the maximum modulus principle applied to the function $e^{f(w)-(\log 4) g(w)}$ on the rectangle with corners at $\pm \frac{1}{2}$ and $\pm \frac{1}{2}+2 i$, it suffices to check (4.31) on the real axis and for $\Re(w)=\frac{1}{2}$. On the real axis we calculate the integral explicitly and verify the inequality using calculus. For $\Re(w)=\frac{1}{2}$ the inequality is strict, so we may verify it computationally for $0 \leq \Im(w) \leq 2$.

Lemma 4.4. For $\sigma>\theta+1$, define

$$
z_{\theta}(\sigma):=\left(\frac{\zeta(2 \sigma+2 \theta) \zeta(2 \sigma-2 \theta)}{\zeta(\sigma+\theta) \zeta(\sigma-\theta)}\right)^{1 / 2} \quad \text { and } \quad Z_{\theta}(\sigma):=(\zeta(\sigma+\theta) \zeta(\sigma-\theta))^{1 / 2} \text {. }
$$

Then

$$
r \frac{z_{\theta}^{\prime}}{z_{\theta}}(\sigma) \geq \Re \frac{L^{\prime}}{L}(\sigma+i t) \geq r \frac{Z_{\theta}^{\prime}}{Z_{\theta}}(\sigma)
$$

and

$$
z_{\theta}(\sigma)^{r} \leq|L(\sigma+i t)| \leq Z_{\theta}(\sigma)^{r}
$$

Proof. From (1.1) we have

$$
r \frac{Z_{\theta}^{\prime}}{Z_{\theta}}(\sigma)-\Re \frac{L^{\prime}}{L}(\sigma+i t)=\sum_{p} \sum_{k=1}^{\infty} p^{-k \sigma} \log p\left(\Re \sum_{j=1}^{r}\left(\alpha_{p, j} p^{-i t}\right)^{k}-\frac{r}{2}\left(p^{k \theta}+p^{-k \theta}\right)\right) .
$$

Pairing the terms for $\alpha_{p, j}$ and $\alpha_{p, j}^{\prime}$, we see that each summand is $\leq 0$, from which the second inequality of (4.33) follows. The first inequality is similar. For (4.34), integrate (4.33) from $\sigma$ to $\infty$.

Theorem 4.5. Suppose $t_{1}$ and $t_{2}$ satisfy (4.19), and set

$$
c_{\theta}:=\log Z_{\theta}\left(\frac{3}{2}\right)+\int_{3 / 2}^{\infty} \log \frac{Z_{\theta}(\sigma)}{z_{\theta}(\sigma)} d \sigma-\int_{3 / 2}^{5 / 2} \log z_{\theta}(\sigma) d \sigma+(\log 4) \frac{z_{\theta}^{\prime}}{z_{\theta}}\left(\frac{3}{2}\right),
$$


where $z_{\theta}$ and $Z_{\theta}$ are as in Lemma 4.4. (In particular, $c_{0} \lesssim 5.65055$.) Then

$(4.37)$

$$
\pi \int_{t_{1}}^{t_{2}} S(t) d t \leq \frac{1}{4} \log \left|Q\left(\frac{3}{2}+i t_{2}\right)\right|+\left(\log 2-\frac{1}{2}\right) \log \left|Q\left(\frac{3}{2}+i t_{1}\right)\right|+c_{\theta} r+\frac{r}{\sqrt{2}(X-5)} .
$$

Remark. Note that there is no assumption on the order of $t_{1}$ and $t_{2}$, so one obtains a lower bound as well by reversing their roles.

Proof. By (4.10), we need upper and lower bounds for $\int_{1 / 2}^{\infty} \log |L(\sigma+i t)| d \sigma$. For the upper bound, we use Lemma 4.1 .

$$
\begin{aligned}
\int_{1 / 2}^{\infty} \log |L(\sigma+i t)| & d \sigma=\int_{1 / 2}^{3 / 2} \log |L(\sigma+i t)| d \sigma+\int_{3 / 2}^{\infty} \log |L(\sigma+i t)| d \sigma \\
\leq & \frac{1}{2} \log B+\frac{1}{2} \int_{1 / 2}^{3 / 2} \log \left|\frac{\gamma(1-\sigma+i t) Q(\sigma+i t)}{\gamma(\sigma+i t)}\right| d \sigma \\
+ & \frac{1}{2} \int_{1 / 2}^{3 / 2} \log \left|\frac{P(\sigma+1+i t)^{2} P(\sigma-2+i t)}{P(\sigma+i t)^{2} P(\sigma-1+i t)}\right| d \sigma+\int_{3 / 2}^{\infty} \log |L(\sigma+i t)| d \sigma .
\end{aligned}
$$

We bound the first and last terms with Lemma 4.4 .

$$
\sup _{\Re(s)=3 / 2}|L(s)|+\int_{3 / 2}^{\infty} \log |L(\sigma+i t)| d \sigma \leq \log Z_{\theta}\left(\frac{3}{2}\right)+\int_{3 / 2}^{\infty} \log Z_{\theta}(\sigma) d \sigma .
$$

For the second term, we replace $\sigma$ by $2-\sigma$ in the top $\gamma$ factor, and use the recurrence for $\gamma$ to get

$$
\frac{1}{2} \int_{1 / 2}^{3 / 2} \log \left|\frac{\gamma(\sigma+1+i t)}{\gamma(\sigma+i t)} \frac{Q(\sigma+i t)}{Q(\sigma-1+i t)}\right| d \sigma .
$$

By the mean value theorem, the integral equals $\Re\left[\frac{\gamma^{\prime}}{\gamma}\left(\sigma^{*}+i t\right)+\frac{Q^{\prime}}{Q}\left(\sigma^{*}-1+i t\right)\right]$ for some $\sigma^{*} \in[1 / 2,5 / 2]$. Thus, by Lemma 4.2, (4.40) is at most

$$
\frac{1}{4} \log \left|Q\left(\frac{3}{2}+i t\right)\right|+r\left(\frac{2}{\pi^{2} X^{2}}+\frac{1}{2 \sqrt{2} X}\right) \text {. }
$$

Similarly, we see that the third term is bounded by $\frac{5 m}{2 X^{2}}$.

We now turn to the lower bound. This part is more delicate since we must take into account the contribution of zeros near $\frac{1}{2}+i t$. We use Turing's idea of comparing $\log |L(s)|$ to $\log |L(s+1)|$; the difference between these looks like a value of the logarithmic derivative, which we can make precise with the help of Lemma 4.3 .

Proceeding, we first clear the poles of $\Lambda$ by writing $F(s)=\Lambda(s) P(s) P(s-1)$. This function then has the Weierstrass-Hadamard product

$$
F(s)=e^{a s+b} \prod_{\rho}\left(1-\frac{s}{\rho}\right) e^{s / \rho},
$$


where $\rho$ runs over the zeros of $\Lambda$ and $\Re(a)=-\sum_{\rho} \Re\left(\frac{1}{\rho}\right)$. Next, we split the integral as follows:

$$
\begin{aligned}
& \int_{1 / 2}^{\infty} \log |L(\sigma+i t)| d \sigma=\int_{1 / 2}^{3 / 2} \log \left|\frac{F(\sigma+i t)}{F(\sigma+1+i t)}\right| d \sigma+\int_{1 / 2}^{3 / 2} \log \left|\frac{\gamma(\sigma+1+i t)}{\gamma(\sigma+i t)}\right| d \sigma \\
& \quad+\int_{1 / 2}^{3 / 2} \log \left|\frac{P(\sigma+1+i t)}{P(\sigma-1+i t)}\right| d \sigma+\int_{3 / 2}^{5 / 2} \log |L(\sigma+i t)| d \sigma+\int_{3 / 2}^{\infty} \log |L(\sigma+i t)| d \sigma .
\end{aligned}
$$

The second term may be estimated, as above, by Lemma 4.2 and the mean value theorem:

$$
\int_{1 / 2}^{3 / 2} \log \left|\frac{\gamma(\sigma+1+i t)}{\gamma(\sigma+i t)}\right| d \sigma \geq \frac{1}{2} \log \left|Q\left(\frac{3}{2}+i t\right)\right|-r\left(\frac{1}{2 \sqrt{2} X}+\frac{4 / \pi^{2}+1 / 4}{X^{2}}\right) .
$$

The third term is positive since $\left|\frac{P(\sigma+1+i t)}{P(\sigma-1+i t)}\right| \geq 1$ for $\sigma \geq 0$. The fourth and fifth terms are handled by Lemma 4.4 .

As for the first term, from (4.42) we have

$$
\log \left|\frac{F(s)}{F(s+1)}\right|=\sum_{\rho} \log \left|\frac{1-\frac{s}{\rho}}{1-\frac{s+1}{\rho}}\right|=-\sum_{\rho} \log \left|\frac{s+1-\rho}{s-\rho}\right| .
$$

Thus,

$$
\int_{1 / 2}^{3 / 2} \log \left|\frac{F(\sigma+i t)}{F(\sigma+i t+1)}\right| d \sigma=-\sum_{\rho} \int_{1 / 2}^{3 / 2} \log \left|\frac{\sigma+i t+1-\rho}{\sigma+i t-\rho}\right| d \sigma .
$$

Now, by the functional equation, the zeros of $\Lambda$ either lie on the line $\Re(s)=\frac{1}{2}$ or come in pairs $\rho, 1-\bar{\rho}$. Applying Lemma 4.3 with $w=\frac{1}{2}+i t-\rho$, we see that (4.46) is bounded below by

$$
-(\log 4) \sum_{\rho} \Re \frac{1}{3 / 2+i t-\rho}
$$

Again by (4.42), this equals

$$
\begin{aligned}
-(\log 4) \Re \frac{F^{\prime}}{F} & \left(\frac{3}{2}+i t\right) \\
& =-(\log 4) \Re\left[\frac{\gamma^{\prime}}{\gamma}\left(\frac{3}{2}+i t\right)+\frac{L^{\prime}}{L}\left(\frac{3}{2}+i t\right)+\frac{P^{\prime}}{P}\left(\frac{3}{2}+i t\right)+\frac{P^{\prime}}{P}\left(\frac{1}{2}+i t\right)\right] \\
& \geq-(\log 4)\left[\frac{1}{2} \log \left|Q\left(\frac{3}{2}+i t\right)\right|+\frac{4 r}{\pi^{2} X^{2}}+r \frac{z_{\theta}^{\prime}}{z_{\theta}}\left(\frac{3}{2}\right)+\frac{2 m}{X^{2}}\right] .
\end{aligned}
$$


Altogether, we get

$$
\begin{aligned}
\int_{1 / 2}^{\infty} \log |L(\sigma+i t)| d \sigma & \geq\left(\frac{1}{2}-\log 2\right) \log \left|Q\left(\frac{3}{2}+i t\right)\right| \\
& +r\left[\int_{3 / 2}^{5 / 2} \log z_{\theta}(\sigma) d \sigma+\int_{3 / 2}^{\infty} \log z_{\theta}(\sigma) d \sigma-(\log 4) \frac{z_{\theta}^{\prime}}{z_{\theta}}\left(\frac{3}{2}\right)\right] \\
& -r\left(\frac{1}{2 \sqrt{2} X}+\frac{\frac{4}{\pi^{2}} \log (4 e)+\frac{1}{4}}{X^{2}}\right)-\frac{4 m \log 2}{X^{2}} .
\end{aligned}
$$

Finally, we combine the upper bound for $t=t_{2}$ and lower bound for $t=t_{1}$. We get the stated main term plus error

$$
r\left(\frac{1}{\sqrt{2} X}+\frac{\frac{2}{\pi^{2}} \log \left(16 e^{3}\right)+\frac{1}{4}}{X^{2}}\right)+m \frac{4(\log 2)+5 / 2}{X^{2}}<\frac{r}{\sqrt{2}(X-5)} .
$$

\section{Rigorous Computation of $L$-Functions}

The methods of Section 4 depend on a fast, rigorous algorithm for evaluating $\Lambda(s)$. We describe one such algorithm, based on the Fast Fourier Transform, in this section. We note that in the case of the Riemann zeta function, a similar technique was developed and used by Odlyzko and Schönhage OS88.

Some algorithms for computing general $L$-functions were described by Dokchitser Dok04. and Rubinstein Rub05. They ultimately boil down to the Cauchy integral formula:

$$
\Lambda\left(s_{0}\right)=\frac{1}{2 \pi i} \int \frac{\gamma(s) L(s)}{s-s_{0}} d s,
$$

where the contour consists of two vertical lines enclosing $s_{0}$. Writing $L(s)$ as a Dirichlet series and using the functional equation, one is lead to study integrals of the form

$$
\frac{1}{2 \pi i} \int \frac{\gamma(s) n^{-s}}{s-s_{0}} d s
$$

taken along a vertical line far to the right. Rubinstein, following an idea of Lagarias and Odlyzko [LO79], inserts a factor designed to cancel the decay of the $\gamma$ factor, e.g. $e^{i \frac{\pi r}{4} \eta s}$ for some $\eta$ close to \pm 1 . Without this factor, very high precision is required to calculate $\Lambda(s)$ when $\Im(s)$ is large.

These algorithms are good when one is interested in computing $\Lambda(s)$ at specific points, e.g. for locating zeros of $L(s)$ precisely. They suffer from the disadvantage of being difficult to carry out rigorously, basically because (5.2) is a two parameter family (indexed by $s_{0}$ and $n$ ) of integrals, for which uniform asymptotics are hard to obtain in certain transition ranges. 
For Turing's method, we need an algorithm for rigorously computing $\Lambda(s)$ for many values of $s$, not necessarily with high precision. For that we consider instead the one parameter integrals

$$
\frac{1}{2 \pi i} \int \Lambda(s) e^{-z s} d s \text { and } \frac{1}{2 \pi i} \int \gamma(s) e^{-z s} d s .
$$

These are essentially Fourier transforms, and they contain enough information for evaluating $\Lambda(s)$ quickly, if one is interested in many points. They also involve only a single Mellin transform, making rigorous computation more accessible.

Precisely, let $\eta \in(-1,1)$ and set $F(t):=\Lambda\left(\frac{1}{2}+i t\right) e^{\frac{\pi r}{4} \eta t}$. Then the (inverse) Fourier transform of $F$ is

$$
\begin{aligned}
\hat{F}(x) & :=\frac{1}{2 \pi} \int_{-\infty}^{\infty} F(t) e^{-i x t} d t=\frac{1}{2 \pi i} \int_{\Re(s)=\frac{1}{2}} \Lambda(s) e^{\left(x+i \frac{\pi r}{4} \eta\right)(1 / 2-s)} d s \\
& =\frac{1}{2 \pi i} \int_{\Re(s)=2} \Lambda(s) e^{\left(x+i \frac{\pi r}{4} \eta\right)(1 / 2-s)} d s-\sum_{\rho \in\left\{1+\lambda_{k}: 1 \leq k \leq m\right\}} \operatorname{Res}_{s=\rho} \Lambda(s) e^{\left(x+i \frac{\pi r}{4} \eta\right)(1 / 2-s)} .
\end{aligned}
$$

The residue sum is straightforward to evaluate assuming we have complete information on any poles of $L(s)$. We multiply the Euler product (1.1) out to a Dirichlet series, writing $L(s)=\sum_{n=1}^{\infty} a_{n} n^{-s}$. Then the first term of (5.4) is

$$
\sum_{n=1}^{\infty} a_{n} \frac{1}{2 \pi i} \int_{\Re(s)=2} \gamma(s) e^{\left(x+i \frac{\pi r}{4} \eta\right)(1 / 2-s)} n^{-s} d s=\epsilon \sum_{n=1}^{\infty} \frac{a_{n}}{\sqrt{n}} G\left(x+\log \frac{n}{\sqrt{N}} ; \eta,\left\{\mu_{j}\right\}\right),
$$

where

$$
G\left(u ; \eta,\left\{\mu_{j}\right\}\right):=\frac{1}{2 \pi i} \int_{\Re(s)=2} e^{\left(u+i \frac{\pi r}{4} \eta\right)(1 / 2-s)} \prod_{j=1}^{r} \Gamma_{\mathbb{R}}\left(s+\mu_{j}\right) d s .
$$

Let us assume for now that we have a procedure to compute $G\left(u ; \eta,\left\{\mu_{j}\right\}\right)$, and thereby $\hat{F}(x)$, to prescribed precision; we return to this point in Section 5.1 below. In order to use the FFT to compute $F$ from $\hat{F}$, we first need to discretize the problem. To that end, let $A, B>0$ be parameters such that $q=A B$ is an integer. By the Poisson summation formula,

$\sum_{k \in \mathbb{Z}} F\left(\frac{m}{A}+k B\right)=\frac{2 \pi}{B} \sum_{k \in \mathbb{Z}} \hat{F}\left(\frac{2 \pi k}{B}\right) e\left(\frac{k m}{A B}\right)=\frac{2 \pi}{B} \sum_{n(\bmod q)} e\left(\frac{m n}{q}\right) \sum_{k \in \mathbb{Z}} \hat{F}\left(\frac{2 \pi n}{B}+2 \pi A k\right)$.

Thus, the functions $\widetilde{F}(m):=\sum_{k \in \mathbb{Z}} F\left(\frac{m}{A}+k B\right)$ and $\widetilde{\hat{F}}(n):=\sum_{k \in \mathbb{Z}} \hat{F}\left(\frac{2 \pi n}{B}+2 \pi A k\right)$, which are periodic in $m, n$ with period $q$, form a discrete Fourier transform pair. 
Note that since $F$ is real-valued, $\hat{F}(-x)=\overline{\hat{F}(x)}$. Thus, for $|n| \leq q / 2$ we have

$$
\tilde{\hat{F}}(n)=\hat{F}\left(\frac{2 \pi n}{B}\right)+\sum_{k=1}^{\infty} \hat{F}\left(\frac{2 \pi n}{B}+2 \pi k A\right)+\sum_{k=1}^{\infty} \overline{\hat{F}\left(-\frac{2 \pi n}{B}+2 \pi k A\right)} .
$$

For $A$ even moderately large, the terms for $k \geq 1$ fall within the asymptotic range. Precise bounds are given in Section [5.3 below; in particular, we may apply Lemma 5.4 with $x=$ $2 \pi\left(A \pm \frac{n}{B}\right)$ to compute the sums over $k$. Hence, it suffices to calculate $\hat{F}(2 \pi n / B)$ for $0 \leq n \leq q / 2$. On the other hand, to compute $F(m / A)$, we need to bound the terms of $\widetilde{F}(m)$ for $k \neq 0$. We have already obtained a suitable bound for the $L$-function in Lemma 4.1. The sum of this bound over $k \neq 0$ is the content of Lemma 5.5.

5.1. Computing $G\left(u ; \eta,\left\{\mu_{j}\right\}\right)$. For brevity, some of the results of this section are only sketched. Our emphasis is on the details necessary for rigorous computation. For more general background information we refer the reader to [Boo03a, Dok04, Rub05].

One simple method for calculating integrals such as (5.6) that is easy to make rigorous is the power and log series, obtained by shifting the contour of (5.6) to the left:

$$
\begin{aligned}
G\left(u ; \eta,\left\{\mu_{j}\right\}\right) & =\sum_{\rho \in \mathbb{C}} \operatorname{Res}_{s=\rho}\left(e^{\left(u+i \frac{\pi r}{4} \eta\right)(1 / 2-s)} \prod_{j=1}^{r} \Gamma_{\mathbb{R}}\left(s+\mu_{j}\right)\right) \\
& =\sum_{\text {poles } \rho} P\left(u ; \rho, \eta,\left\{\mu_{j}\right\}\right) e^{(1 / 2-\rho) u}
\end{aligned}
$$

where $P\left(u ; \rho, \eta,\left\{\mu_{j}\right\}\right)$ is a polynomial of degree one less than the order of the pole at $\rho$.

For example, in the case of Galois representations, the $\mu_{j}$ are all either 0 or 1 , and the residues in (5.9) may be evaluated by the following:

$$
\begin{aligned}
\Gamma_{\mathbb{R}}(s)=\frac{2}{s+2 k} \frac{(-2 \pi)^{k}}{(2 k) ! !} \exp [ & \left(\sum_{n=1}^{k} \frac{1}{2 n}-\frac{1}{2} \log \left(\pi e^{\gamma}\right)\right)(s+2 k) \\
& \left.+\sum_{j=2}^{\infty} \frac{1}{j}\left((-1)^{j} 2^{-j} \zeta(j)+\sum_{n=1}^{k} \frac{1}{(2 n)^{j}}\right)(s+2 k)^{j}\right] \\
=\frac{(-2 \pi)^{k}}{(2 k-1) ! !} \exp [ & {\left[\sum_{n=1}^{k} \frac{1}{2 n-1}-\frac{1}{2} \log \left(4 \pi e^{\gamma}\right)\right)(s+2 k-1) } \\
& \left.+\sum_{j=2}^{\infty} \frac{1}{j}\left((-1)^{j}\left(1-2^{-j}\right) \zeta(j)+\sum_{n=1}^{k} \frac{1}{(2 n-1)^{j}}\right)(s+2 k-1)^{j}\right],
\end{aligned}
$$

for any integer $k \geq 0$. For general $\mu_{j}$, we need an algorithm to calculate the values of $\Gamma$ and its derivatives at an arbitrary point in the complex plane; we assume without further comment that this is available when necessary. 
We say that $\mu_{j}$ and $\mu_{k}$ are equivalent if $\mu_{j}-\mu_{k} \in 2 \mathbb{Z}$. For $\mu$ ranging over an equivalence class, the functions $\Gamma_{\mathbb{R}}(s+\mu)$ share all but finitely many poles. Thus, (5.9) may be broken naturally into parts corresponding to each class. We can bound the tail of each part as follows.

Lemma 5.1. Let $\rho$ be a pole of $g(s)=e^{\left(u+i \frac{\pi r}{4} \eta\right)(1 / 2-s)} \prod_{j=1}^{r} \Gamma_{\mathbb{R}}\left(s+\mu_{j}\right)$ of order $n$, with $\Re\left(\rho+\mu_{j}\right) \leq 0$ for $j=1, \ldots, r$ and $(2 \pi)^{r} e^{2 u}<\frac{1}{2} \prod_{j=1}^{r}\left(\left|2-\rho-\mu_{j}\right|-1\right)$. Let $c_{j}$ be the coefficients of the polar part of $g$ around $\rho$, i.e. such that $g(s+\rho)-\sum_{j=1}^{n} c_{j} s^{-j}$ is holomorphic at $s=0$. Then

$$
\left|\sum_{k=1}^{\infty} \operatorname{Res}_{s=\rho-2 k} g(s)\right|<\max \left|c_{j}\right| .
$$

Proof. First note that

$$
\begin{aligned}
g(s+\rho-2) & =(2 \pi)^{r} e^{2 u+i \frac{\pi r}{2} \eta} g(s+\rho) \prod_{j=1}^{r}\left(s+\rho-2+\mu_{j}\right)^{-1} \\
& =\frac{(-2 \pi)^{r} e^{2 u+i \frac{\pi r}{2} \eta}}{\prod_{j=1}^{r}\left(2-\rho-\mu_{j}\right)} \cdot \frac{g(s+\rho)}{\prod_{j=1}^{r}\left(1-\frac{s}{2-\rho-\mu_{j}}\right)} .
\end{aligned}
$$

Next, let $f(s)$ be a meromorphic function with polar part $a_{1} s^{-1}+\ldots+a_{n} s^{-n}$ at 0 . If $x$ is a complex number with $|x|<1$ then the function $\frac{f(s)}{1-x s}$ has polar part $a_{1}^{\prime} s^{-1}+\ldots+a_{n}^{\prime} s^{-n}$, where $a_{j}^{\prime}=\sum_{k=0}^{n-j} a_{j+k} x^{k}$. Thus,

$$
\max \left|a_{j}^{\prime}\right| \leq \frac{\max \left|a_{j}\right|}{1-|x|}
$$

Let $c_{1}^{\prime} s^{-1}+\ldots+c_{n}^{\prime} s^{-n}$ be the polar part of $g(s+\rho-2)$. Applying (5.13) $r$ times, we see from (5.12) that

$$
\begin{aligned}
\max \left|c_{j}^{\prime}\right| & \leq \frac{(2 \pi)^{r} e^{2 u}}{\prod_{j=1}^{r}\left|2-\rho-\mu_{j}\right|} \cdot \frac{\max \left|c_{j}\right|}{\prod_{j=1}^{r}\left(1-\frac{1}{\left|2-\rho-\mu_{j}\right|}\right)} \\
& =\frac{(2 \pi)^{r} e^{2 u} \max \left|c_{j}\right|}{\prod_{j=1}^{r}\left(\left|2-\rho-\mu_{j}\right|-1\right)}<\frac{1}{2} \max \left|c_{j}\right| .
\end{aligned}
$$

Repeating this procedure, we see that the coefficients of the polar part of $g(s+\rho-2 k)$ are $<2^{-k} \max \left|c_{j}\right|$. The conclusion follows.

The lemma says roughly that if we compute the residue sum for all poles with real part down to $\Re(\rho)$, the tail of the series (from poles at $\rho-2 k$ ) may be bounded by the data from the last term added. Moreover, (5.12) gives an algorithm for computing the data at $\rho-2$ from that at $\rho$, and shows that the terms eventually decrease factorially. Thus, we may use this to compute $G\left(u ; \eta,\left\{\mu_{j}\right\}\right)$ to any desired precision for a given $u$. 
Since the $\mu_{j}$ are arbitrary, this procedure is general enough to compute derivatives of $G\left(u ; \eta,\left\{\mu_{j}\right\}\right)$ as well. For instance, for any $k$ we have

$$
G^{\prime}\left(u ; \eta,\left\{\mu_{j}\right\}\right)=\left(\mu_{k}+1 / 2\right) G\left(u ; \eta,\left\{\mu_{j}\right\}\right)-2 \pi G\left(u ; \eta,\left\{\mu_{j}^{\prime}\right\}\right)
$$

where $\mu_{j}^{\prime}=\mu_{j}$ if $j \neq k$ and $\mu_{k}^{\prime}=\mu_{k}+2$. Higher derivatives may be computed in a similar fashion. (In fact $G$ satisfies an $r$ th order differential equation, due to the recurrence for $\Gamma$; thus, the derivatives of all orders are determined from the first $r$.)

Note that for $u$ large this method requires high precision due to cancellation, and is therefore inefficient. The essential point that makes it worthwhile is that for a given $\gamma$ factor the calculations need only be performed once, as one can develop local approximations to $G\left(u ; \eta,\left\{\mu_{j}\right\}\right)$ for later rapid evaluation. The computation may then be recycled and used for any $L$-function with the same $\mu_{j}$; this is useful for functions in an arithmetic family, such as Artin $L$-functions.

More precisely, suppose we wish to calculate $G\left(u ; \eta,\left\{\mu_{j}\right\}\right)$ for $u$ in an interval $I$. Choose $\varepsilon>0$ and sample points $u_{m}$ such that each $u \in I$ is contained in a unique interval $\left[u_{m}-\varepsilon, u_{m}+\varepsilon\right)$. For $u$ in the $m$ th interval, we have by Taylor's theorem

$$
G\left(u ; \eta,\left\{\mu_{j}\right\}\right)=\sum_{k=0}^{K-1} \frac{G^{(k)}\left(u_{m} ; \eta,\left\{\mu_{j}\right\}\right)}{k !}\left(u-u_{m}\right)^{k}+\Theta\left(\max _{\left|u^{*}-u_{m}\right| \leq \varepsilon} \frac{\left|G^{(K)}\left(u^{*} ; \eta,\left\{\mu_{j}\right\}\right)\right|}{K !} \varepsilon^{K}\right) .
$$

We may evaluate the derivatives precisely using (5.9). As for the $K$ th derivative, a uniform bound is obtained by shifting the contour of (5.6) to $\Re(s)=\frac{1}{2}$ :

$$
\frac{\left|G^{(K)}\left(u ; \eta,\left\{\mu_{j}\right\}\right)\right|}{K !} \leq \frac{1}{2 \pi} \int_{-\infty}^{\infty} \frac{|t|^{K}}{K !} e^{\frac{\pi r}{4} \eta t} \prod_{j=1}^{r}\left|\Gamma_{\mathbb{R}}\left(\frac{1}{2}+i t+\mu_{j}\right)\right| d t
$$

For large $K$ this is of size $\left(\frac{4}{\pi r(1-\eta)}\right)^{K}$; thus as long as $\varepsilon$ is small compared to $\frac{\pi r}{4}(1-\eta)$, we may compute and store the coefficients of (5.16), yielding a fast method to calculate $G\left(u ; \eta,\left\{\mu_{j}\right\}\right)$ for any $u \in I$.

Moreover, we can improve the efficiency of our algorithm if the sample points of (5.8) coincide with multiplies of $2 \varepsilon$, i.e. if $\frac{\pi}{\varepsilon B} \in \mathbb{Z}$. For any given sample point $x$, we approximate $\hat{F}(x)$ via a truncated series (keeping track of the error terms from (5.16) and Lemma 5.3 
below):

$$
\begin{aligned}
& \sum_{n=1}^{M} \frac{a_{n}}{\sqrt{n}} G\left(x+\log \frac{n}{\sqrt{N}} ; \eta,\left\{\mu_{j}\right\}\right) \\
& \quad \approx \sum_{m} \sum_{\log \frac{n}{\sqrt{N}} \in\left[u_{m}-\varepsilon, u_{m}+\varepsilon\right)} \frac{a_{n}}{\sqrt{n}} \sum_{k=0}^{K-1} \frac{G^{(k)}\left(x+u_{m} ; \eta,\left\{\mu_{j}\right\}\right)}{k !}\left(\log \frac{n}{\sqrt{N}}-u_{m}\right)^{k} \\
& \quad=\sum_{k=0}^{K-1} \sum_{m} \frac{G^{(k)}\left(x+u_{m} ; \eta,\left\{\mu_{j}\right\}\right)}{k !} S_{m}^{(k)}
\end{aligned}
$$

where

$$
S_{m}^{(k)}:=\sum_{\log \frac{n}{\sqrt{N}} \in\left[u_{m}-\varepsilon, u_{m}+\varepsilon\right)} \frac{a_{n}}{\sqrt{n}}\left(\log \frac{n}{\sqrt{N}}-u_{m}\right)^{k} .
$$

Since $x+u_{m}$ is another sample point $u_{m^{\prime}}$, the $k$ th term of (5.18) is a convolution of the sequences (indexed by $m) \frac{G^{(k)}\left(u_{m} ; \eta,\left\{\mu_{j}\right\}\right)}{k !}$ and $S_{m}^{(k)}$; thus, we may evaluate it efficiently for all $x$ simultaneously by appealing again to the FFT.

5.2. Complexity. We may now consider the complexity of the algorithm. Note that by Stirling's formula, $F(t)$ decays roughly like $e^{-(1-\eta) \frac{\pi r}{4} t}$ for $t>0$. Ideally we should choose $1-\eta$ of size $T^{-1}$ in order to compute values up to height $T$. Adjusting the constant of proportionality (i.e. choosing $\eta$ relatively close to or far from 1 ) allows us to trade off the computational precision and number of coefficients needed to overcome the error terms below. Finding a good compromise between these two is best done by trial and error; cf. Section 6.

Since $\delta \asymp T^{-1}$, Lemma [5.3 shows that in order to compute (5.18) we need on the order of $\sqrt{N T^{r}}$ terms of (5.5) , or roughly the square root of the analytic conductor. Note that the values of $A$ and $B$ enter only in the Fourier transforms, and do not significantly affect the computation of (5.18). We set $B$ equal to a multiple of $T$, depending on the chosen value of $\eta$. As for $A$, as mentioned in Section 3 , the density of zeros of $F(t)$ around height $T$ is $\frac{1}{2 \pi} \log N\left(\frac{T}{2 \pi}\right)^{r}$; one can expect to take $A$ equal to a multiple of this. Thus, this method has complexity consistent with computing a single value by the approximate functional equation, after which we get many values in mean time $O_{\varepsilon}\left((N T)^{\varepsilon}\right)$, which is essentially best possible. The gain comes from the fact, as emphasized above, that only a single $G$-function is involved.

5.3. Asymptotics. To complete our understanding of $G\left(u ; \eta,\left\{\mu_{j}\right\}\right)$, in order to accurately calculate (5.5), we need an asymptotic bound for large $u$. If we write $\mu=-\frac{1}{2}+\frac{1}{r}(1+$ 
$\left.\sum_{j=1}^{r} \mu_{j}\right)$ then by the method of stationary phase, we have

$$
G\left(u ; \eta,\left\{\mu_{j}\right\}\right)=\sqrt{\frac{2^{r+1}}{r}} e^{\mu\left(u+i \frac{\pi r}{4} \eta\right)} \exp \left(-e^{\frac{2}{r}\left(u+i \frac{\pi r}{4} \eta\right)}\right)\left(1+O\left(e^{-2 u / r}\right)\right),
$$

where the implied constant depends on the $\mu_{j}$. For $r=1$ the formula is exact, i.e. the $O$ term is 0 . For $r>1$, one can work out explicit constants case by case, which is preferable if sharp error terms are desired. Otherwise, we get a bound that is close to (5.20) simply by shifting the contour of (5.6) to the right.

Lemma 5.2. Let $\delta=\frac{\pi}{2}(1-|\eta|), \nu_{j}=\frac{\Re\left(\mu_{j}\right)-1}{2}+\frac{1}{2 r}, \mu=-\frac{1}{2}+\frac{1}{r}\left(1+\sum_{j=1}^{r} \mu_{j}\right), K=$ $2 \sqrt{\frac{2^{r+1}}{r} \frac{e^{\delta(r-1)}}{\delta}} e^{-\frac{\pi r \eta \Im(\mu)}{4}}$, and $X=\pi r \delta e^{-\delta} e^{2 u / r}$. Then for $X \geq r$,

$$
\left|G\left(u ; \eta,\left\{\mu_{j}\right\}\right)\right| \leq K e^{\Re(\mu) u} e^{-X} \prod_{j=1}^{r}\left(1+\frac{r \nu_{j}}{X}\right)^{\nu_{j}}
$$

Remark. This is within a factor $O\left(\delta^{-1 / 2}\right)$ of the correct asymptotic if $\delta \ll r e^{-u / r}$.

Proof. We write $s=2 \sigma+2 i t$ in (5.6) to get

$\left|G\left(u ; \eta,\left\{\mu_{j}\right\}\right)\right| \leq \pi^{-r\left(\sigma+\frac{\Re(\mu)}{2}+\frac{1}{4}\right)-\frac{1}{2}} e^{u\left(\frac{1}{2}-2 \sigma\right)-\frac{\pi r \eta \Im(\mu)}{4}} \int_{-\infty}^{\infty} \prod_{j=1}^{r}\left|\Gamma\left(\sigma+i t+\frac{\mu_{j}}{2}\right) e^{\frac{\pi \eta}{4}\left(2 t+\Im\left(\mu_{j}\right)\right)}\right| d t$.

Applying Hölder's inequality, we get integrals of the form

$$
\int_{-\infty}^{\infty}|\Gamma(a+i t)|^{r} e^{\frac{\pi r \eta}{2} t} d t
$$

where $a=\sigma+\Re\left(\mu_{j}\right) / 2$. Assuming $\sigma \geq 1$, we may apply the inequality

$$
|\Gamma(a+i t)| \leq \sqrt{2 \pi}(a+|t|)^{a-1 / 2} e^{-\pi|t| / 2} \quad \text { for } a \geq \frac{1}{2}
$$

(To see this, note that $\left|\Gamma\left(\frac{1}{2}+i t\right)\right|=\sqrt{\pi \operatorname{sech} \pi t}$, use the recurrence for $\Gamma$ and (4.24) to reduce to the region $\frac{1}{2} \leq a \leq \frac{3}{2}, 0 \leq t \leq 2$, where the inequality may be checked computationally.) Thus, we have

$$
\begin{aligned}
\int_{-\infty}^{\infty}|\Gamma(a+i t)|^{r} e^{\frac{\pi r \eta}{2} t} d t & \leq 2(2 \pi)^{r / 2} \int_{0}^{\infty}(a+t)^{r(a-1 / 2)} e^{-r \delta t} d t \\
& \leq 2(2 \pi)^{r / 2} e^{\delta r a} \frac{\Gamma(r(a-1 / 2)+1)}{(\delta r)^{r(a-1 / 2)+1}} \\
& \leq 2(2 \pi)^{\frac{r+1}{2}} \sqrt{\frac{e^{\delta(r-1)}}{\delta r}}\left(\frac{e^{\delta}}{\delta} \frac{a-1 / 2+1 / 2 r}{e}\right)^{r(a-1 / 2)+1 / 2}
\end{aligned}
$$


Substituting this bound into (5.22) and collecting terms we obtain

$$
K e^{\Re(\mu) u} \prod_{j=1}^{r}\left(\frac{\sigma+\nu_{j}}{e X / r}\right)^{\sigma+\nu_{j}} \leq K e^{\Re(\mu) u} e^{-\sigma r} \prod_{j=1}^{r}\left(\frac{r \sigma}{X}\right)^{\sigma+\nu_{j}}\left(1+\frac{\nu_{j}}{\sigma}\right)^{\nu_{j}} .
$$

The result follows upon taking $\sigma=X / r$.

With this bound in hand, we can estimate the error in truncating the series (5.5).

Lemma 5.3. Let $M$ be a positive integer, $x \in \mathbb{R}$. Let $\delta, \nu_{j}, \mu, K$ be as in Lemma 5.2 and set $X=\pi r \delta e^{-\delta}\left(e^{x} / \sqrt{N}\right)^{2 / r}$. Let $C, \alpha \geq 0$ be such that $\left|a_{n}\right| \leq C n^{\alpha}$ for all $n$, and put $c=\Re(\mu)+\frac{1}{2}+\alpha, c^{\prime}=\max (c r / 2-1,0)$. Then for $X M^{2 / r}>\max \left(c^{\prime}, r\right)$,

$$
\begin{aligned}
& \left|\sum_{n>M} \frac{a_{n}}{\sqrt{n}} G\left(x+\log \frac{n}{\sqrt{N}} ; \eta,\left\{\mu_{j}\right\}\right)\right| \\
& \quad \leq \frac{K r}{2}\left(\frac{e^{x}}{\sqrt{N}}\right)^{\Re(\mu)} \frac{C M^{c} e^{-X M^{2 / r}}}{X M^{2 / r}-c^{\prime}} \prod_{j=1}^{r}\left(1+\frac{r \nu_{j}}{X M^{2 / r}}\right)^{\nu_{j}} .
\end{aligned}
$$

Remark. Different values of $C$ and $\alpha$ are appropriate for different ranges. For small $M$, one can take $C=1, \alpha=\log _{2} r+\theta$, while for larger $M$ it is better to choose a smaller value of $\alpha$ and compute $C$ from the coefficients.

Proof. Using Lemma 5.2, we have

$$
\sum_{n>M} \frac{\left|a_{n}\right|}{\sqrt{n}}\left|G\left(x+\log \frac{n}{\sqrt{N}} ; \eta,\left\{\mu_{j}\right\}\right)\right| \leq K^{\prime} \sum_{n>M} n^{c-1} e^{-X n^{2 / r}}
$$

where

$$
K^{\prime}=C K\left(\frac{e^{x}}{\sqrt{N}}\right)^{\Re(\mu)} \prod_{j=1}^{r}\left(1+\frac{r \nu_{j}}{X M^{2 / r}}\right)^{\nu_{j}}
$$

The condition on $X$ ensures that the terms of (5.28) are monotonically decreasing. Thus, we can estimate by the integral

$$
K^{\prime} \int_{M}^{\infty} t^{c-1} e^{-X t^{2 / r}} d t=\frac{K^{\prime} r}{2}\left(X^{-r / 2}\right)^{c} \int_{X M^{2 / r}}^{\infty} y^{c r / 2-1} e^{-y} d y \leq \frac{K^{\prime} r}{2} \frac{M^{c} e^{-X M^{2 / r}}}{X M^{2 / r}-c^{\prime}}
$$

The next two lemmas bound the error introduced in discretization. 
Lemma 5.4. Let $x \in \mathbb{R}, A \geq \frac{1}{2 \pi}$, and let notation be as in Lemma 5.3. Then for $X>$ $\max \left(c^{\prime}, r\right)$,

$$
\begin{aligned}
\sum_{k=0}^{\infty} \hat{F}(x+2 \pi k A) & =-\sum_{\rho \in\left\{1+\lambda_{k}: 1 \leq k \leq m\right\}} \operatorname{Res}_{s=\rho} \frac{\Lambda(s) e^{\left(x+i \frac{\pi r}{4} \eta\right)(1 / 2-s)}}{1-e^{2 \pi A(1 / 2-s)}} \\
& +\Theta\left[\frac{K}{1-e^{-\pi A}}\left(\frac{e^{x}}{\sqrt{N}}\right)^{\Re(\mu)} e^{-X}\left(1+\frac{C r / 2}{X-c^{\prime}}\right) \prod_{j=1}^{r}\left(1+\frac{r \nu_{j}}{X}\right)^{\nu_{j}}\right] .
\end{aligned}
$$

Proof. The residue sum comes from summing the polar part of (5.4) with $x+k A$ in place of $x$. For the rest, we apply Lemmas 5.2 and 5.3 (with $M=1$ ) to get the bound

$$
K\left(\frac{e^{x}}{\sqrt{N}}\right)^{\Re(\mu)} e^{-X}\left(1+\frac{C r / 2}{X-c^{\prime}}\right) \prod_{j=1}^{r}\left(1+\frac{r \nu_{j}}{X}\right)^{\nu_{j}}
$$

for the $k=0$ term. To pass from this to the $k$ th term, we multiply by a factor not exceeding

$$
\begin{aligned}
e^{2 \pi k A \Re(\mu)} e^{-X(\exp (4 \pi k A / r)-1)} & =\exp \left(2 \pi k A\left[\Re(\mu)-\frac{2 X}{r}-\frac{X}{2 \pi k A}\left(e^{4 \pi k A / r}-1-\frac{4 \pi k A}{r}\right)\right]\right) \\
& \leq \exp \left(-2 \pi k A\left[\alpha+\frac{1}{2}-\frac{2}{r}+\frac{4 \pi k A}{r}\right]\right) \leq e^{-\pi k A} .
\end{aligned}
$$

The result follows on summing the geometric series.

Lemma 5.5. Let $t \in \mathbb{R}$ and put $s=\frac{1}{2}+i t$,

$$
E=Z_{\theta}(3 / 2)^{r}|\gamma(s)| e^{\frac{\pi r}{4} \eta t}\left|Q(s) \frac{P(s+1)^{2} P(s-2)}{P(s)^{2} P(s-1)}\right|^{1 / 2},
$$

and

$$
\beta=\frac{\pi r}{4}-\frac{1}{2} \sum_{j=1}^{r} \arctan \frac{\Re\left(s+\mu_{j}\right)}{\left|\Im\left(s+\mu_{j}\right)\right|}-\frac{4}{\pi^{2}} \sum_{j=1}^{r} \frac{1}{\left|\Im\left(s+\mu_{j}\right)^{2}-\Re\left(s+\mu_{j}\right)^{2}\right|} .
$$

(1) If $\Im\left(s+\mu_{j}\right)>0$ for all $j=1, \ldots, r$ and $\beta-\frac{\pi r}{4} \eta>0$ then

$$
\left|\sum_{k=0}^{\infty} F(t+k B)\right| \leq \frac{E}{1-e^{-\left(\beta-\frac{\pi r}{4} \eta\right) B}} .
$$

(2) If $\Im\left(s+\mu_{j}\right)<0$ for all $j=1, \ldots, r$ and $\beta+\frac{\pi r}{4} \eta>0$ then

$$
\left|\sum_{k=0}^{\infty} F(t-k B)\right| \leq \frac{E}{1-e^{-\left(\beta+\frac{\pi r}{4} \eta\right) B}} .
$$


Proof. We treat only the first case, the second being similar. Lemmas 4.1 and 4.4 imply the bound $|F(t)| \leq E$. We consider the same bound with $t$ replaced by $t+k B$. Note that if $\left|\Im\left(s+\mu_{j}\right)\right|$ increases for all $j$ then the factor involving $P$ is non-increasing. For the $\gamma$ and $Q$ factors, by the mean value theorem we have

$$
\log \left(\left|\frac{\gamma(s+i k B)}{\gamma(s)}\right|\left|\frac{Q(s+i k B)}{Q(s)}\right|^{1 / 2}\right)=-k B \Im\left(\frac{\gamma^{\prime}}{\gamma}\left(s^{*}\right)+\frac{1}{2} \frac{Q^{\prime}}{Q}\left(s^{*}\right)\right)
$$

for some $s^{*}$ on the line between $s$ and $s+i k B$. Using (4.24), this is

$$
\begin{aligned}
-k B & \Im \sum_{j=1}^{r}\left(\frac{1}{2} \log \frac{s^{*}+\mu_{j}}{2}+\Theta\left(\frac{4 / \pi^{2}}{\left|\Im\left(s^{*}+\mu_{j}\right)^{2}-\Re\left(s^{*}+\mu_{j}\right)^{2}\right|}\right)\right) \\
& \leq-k B\left(\frac{\pi r}{4}-\frac{1}{2} \sum_{j=1}^{r} \arctan \frac{\Re\left(s^{*}+\mu_{j}\right)}{\Im\left(s^{*}+\mu_{j}\right)}-\frac{4}{\pi^{2}} \sum_{j=1}^{r} \frac{1}{\left|\Im\left(s^{*}+\mu_{j}\right)^{2}-\Re\left(s^{*}+\mu_{j}\right)^{2}\right|}\right) \\
& \leq-\beta k B .
\end{aligned}
$$

Thus, $|F(t+k B)| \leq E e^{-\left(\beta-\frac{\pi r}{4} \eta\right) k B}$. The conclusion follows.

\section{Numerical RESUlts}

We have applied the methods described in the previous sections to a few examples of splitting fields of polynomials with Galois group $S_{5}$ and $A_{5}$, as listed in Table 6.1 For the $A_{5}$ cases, the Artin conjecture is true for all representations by known cases of functoriality Kim94, JM01. That speeds up the process, since we may apply Turing's method to the Artin $L$-functions directly. For the $S_{5}$ examples we verify both conjectures. As expected, we found no counterexamples to either conjecture in the tested range $|t| \leq 100$.

\begin{tabular}{|r|r|l|}
\hline polynomial & group & splitting field discriminant \\
\hline$x^{5}-68 x-68$ & $S_{5}$ & $2^{96} 3^{60} 17^{96}$ \\
$x^{5}-x^{4}-8 x^{3}+10 x^{2}-x-5$ & $S_{5}$ & $2^{160} 3^{96} 7^{96}$ \\
$x^{5}-x^{4}+3 x^{3}-11 x^{2}-8 x-8$ & $S_{5}$ & $2^{220} 13^{96}$ \\
$x^{5}+2 x^{3}-4 x^{2}-2 x+4$ & $A_{5}$ & $2^{90} 73^{30}$ \\
$x^{5}+20 x+16$ & $A_{5}$ & $2^{90} 5^{78}$ \\
$x^{5}-x^{4}+8 x^{3}-6 x^{2}+14 x-6$ & $A_{5}$ & $2^{90} 193^{30}$ \\
$x^{5}-7 x^{3}-17 x^{2}+18 x+73$ & $A_{5}$ & $2^{40} 487^{30}$ \\
$x^{5}+8 x^{3}+7 x^{2}+172 x+53$ & $A_{5}$ & $2083^{30}$ \\
\hline
\end{tabular}

TABLE 6.1. Tested polynomials

To illustrate the methods, we discuss in detail the $S_{5}$ field of discriminant $2^{96} 3^{60} 17^{96}$ given by the polynomial $f(x)=x^{5}-68(x+1)$. Recall that $S_{5}$ has seven irreducible representations. We label them $1, \chi, \rho_{4}, \rho_{4}^{\prime}=\rho_{4} \otimes \chi, \rho_{5}, \rho_{5}^{\prime}=\rho_{5} \otimes \chi$ and $\rho_{6}$, where 
$\chi$ is the sign character and the subscripts indicate the dimensions. As it will turn out, the limiting factor in our computations is the conductor of $\rho_{6}$, which in our example is $36081072=2^{4} 3^{3} 17^{4}$. This is the smallest among the table of $S_{5}$ polynomials given in [KM]; since that table is ordered by the conductor of $\rho_{4}$, it is likely that smaller examples exist. (We note, however, that if one is interested only in verifying some instances of Artin's conjecture and not the Riemann hypothesis, the holomorphy of $L\left(s, \rho_{5}^{\prime}\right)$ may be checked much more easily; there the limiting factor is the conductor of $\rho_{4}$, of which [KM yields examples as small as 1609 . We have not pursued this possibility.)

Note that $1, \chi$ and $\rho_{6}$ are monomial representations, so Artin's conjecture is true for those. Equation (6.1) below shows that $L\left(s, \rho_{4}\right)$ and $L\left(s, \rho_{5}\right)$ are holomorphic except possibly at the zeros of $\zeta(s)$. Twisting by $\chi$, we see similarly that $L\left(s, \rho_{4}^{\prime}\right)$ and $L\left(s, \rho_{5}^{\prime}\right)$ are holomorphic away from the zeros of $L(s, \chi)$. Moreover, we learn from GAP that the representations $\rho_{4} \oplus \rho_{6}, \rho_{4}^{\prime} \oplus \rho_{6}, \rho_{5} \oplus \rho_{4}^{\prime} \oplus \chi$ and $\rho_{5}^{\prime} \oplus \rho_{4} \oplus 1$ are all monomial. Thus, in order to verify the holomorphy of $L\left(s, \rho_{4}\right), L\left(s, \rho_{4}^{\prime}\right), L\left(s, \rho_{5}\right)$ and $L\left(s, \rho_{5}^{\prime}\right)$ it is enough to check that $L\left(s, \rho_{6}\right)$ and $L\left(s, \rho_{4}^{\prime} \oplus \chi\right)$ are non-vanishing at zeros of $\zeta(s)$ and, similarly, that $L\left(s, \rho_{6}\right)$ and $L\left(s, \rho_{4} \oplus 1\right)$ do not vanish at the zeros of $L(s, \chi)$. Applying Turing's method to these functions as well as $L\left(s, \rho_{5} \oplus 1\right)$ and $L\left(s, \rho_{5}^{\prime} \oplus \chi\right)$, we can deduce both Artin's conjecture and the Riemann hypothesis (up to the tested height) for all representations.

In what follows we describe the numerical procedure in detail for $L\left(s, \rho_{6}\right)$. First we must choose a value of $\eta$ to use for the computation of $G\left(u ; \eta,\left\{\mu_{j}\right\}\right)$. One can aim to limit either the number of Dirichlet coefficients $a_{n}$ or the precision required in the computation. Since the coefficients are relatively easy to compute in our case (we have $2^{32}$ of them), we try for the latter. The largest error comes from Lemma 5.3 with $x=0$, and is of size roughly $M^{c} \exp \left(-\pi r \delta e^{-\delta}(M / \sqrt{N})^{2 / r}\right)$, where $\delta=\frac{\pi}{2}(1-|\eta|), r=6, N=36081072$ and $M=2^{32}$. Examining the local factors at small primes we determine that $\left|a_{n}\right| \leq 1.26 n^{\log _{4243} 6}$, yielding $c=\frac{2}{3}+\log _{4243} 6$. (The $\mu_{j}$ in this case are $0,0,0,1,1,1$.) This error term should be compared to the size of the function being evaluated, which is roughly $e^{-\delta r t / 2}$. From Theorem 4.5 we find that to apply Turing's method up to height $t=100$ we need to be able to compute the $L$-function up to about $t=115$. Trying a few values of $\eta$, we find that with $\eta=0.98$ the error terms are of size $10^{-14}$, compared to $10^{-5}$ for the size of the function. Thus, with this choice we should use a precision of at least 14 digits; in fact we carry out most computations to 30 digits.

All computations were performed on a $3 \mathrm{GHz}$ PC running Linux. They were divided into several steps:

(1) Computing the Dirichlet coefficients $a_{n}$;

(2) Estimating zeros by the explicit formula;

(3) Computing $G^{(k)}\left(u_{m} ; \eta,\left\{\mu_{j}\right\}\right)$;

(4) Computing $S_{m}^{(k)}$;

(5) Computing (5.18) and $L\left(s, \rho_{6}\right)$ by FFT;

(6) Turing's method. 
To ensure correct results, we used the arbitrary precision interval arithmetic package MPFI RR05 for steps 3 through 7 . We discuss the steps in more detail below.

6.1. Computing $a_{n}$. First we consider methods of computing the coefficients of the $L$ functions $L(s, \rho)$ for all irreducible representations $\rho$. One way is to express them as ratios of products of Hecke $L$-functions, as given by Brauer's theorem; in fact for $S_{5}$ we may express each in terms of Dedekind zeta functions of intermediate fields. Precisely, let $k$ be the quadratic extension of $\mathbb{Q}$ associated to $\chi, F=\mathbb{Q}\left(x_{1}\right) \subset M=\mathbb{Q}\left(x_{1}, x_{2}\right)$ where $x_{1}$ and $x_{2}$ are distinct roots of $f$, and $E=\mathbb{Q}(y) \subset K$ where $y$ is a root of the sextic resolvent (a formula for which is given in [Dum91]); then we have

$$
\begin{array}{rlrl}
L(s, \chi) & =\frac{\zeta_{k}(s)}{\zeta(s)}, & L\left(s, \rho_{4}\right)=\frac{\zeta_{F}(s)}{\zeta(s)}, & L\left(s, \rho_{5}\right)=\frac{\zeta_{E}(s)}{\zeta(s)} \\
L\left(s, \rho_{6}\right)=\frac{\zeta_{k}(s) \zeta_{E}(s) \zeta_{M}(s)}{\zeta_{k E}(s) \zeta_{F}(s)^{2}}, & L\left(s, \rho_{4}^{\prime}\right)=\frac{\zeta(s) \zeta_{k F}(s)}{\zeta_{k}(s) \zeta_{F}(s)}, & L\left(s, \rho_{5}^{\prime}\right)=\frac{\zeta(s) \zeta_{k E}(s)}{\zeta_{k}(s) \zeta_{E}(s)}
\end{array}
$$

In turn, we may compute each of the Dedekind zeta functions using the ideal factorization functions built in to PARI The04. This facilitates the computation of local factors at primes dividing the discriminant, allowing us to avoid a detailed study of the possible types of ramification. However, it is not well-suited to working out many coefficients.

Fortunately, there is a faster method that works for all but finitely many primes. Table 6.2 shows the unramified local factors for each representation and conjugacy class (labelled by the order of elements in the class), where we write $x$ for $p^{-s}$. For $S_{5}$ it turns out that the Frobenius conjugacy class at $p$ is determined by the number of linear and quadratic factors of the reduction $\bar{f}$ of $f$ modulo $p$, which may be computed from the degrees of $\operatorname{gcd}\left(x^{p^{n}}-x, \bar{f}(x)\right)$ for $n=1,2$. That computation requires $O(\log p)$ multiplications and additions mod $p$. Thus, by the prime number theorem, for each $L(s, \rho)$ we may determine the Dirichlet coefficients $a_{n}$ for $n \leq X$ in time $O(X)$ (assuming mod $p$ multiplications and additions take bounded time, which is appropriate for numbers of the size that we consider). Up to the implied constant, that is best possible. Moreover, the technique is very fast in practice; we found that it takes approximately seven hours to compute the local factors for all $p<2^{32}$.

6.2. Estimating zeros. With our computed coefficients, we readily obtain estimates for the low zeros by the method of Section 3. Figure 6.1 shows graphs of $F_{X}(t)$, with $X=$ $\log \left(2^{32}\right)$, for each irreducible $L$-function. The spikes correspond to zeros, from which we get the estimates for the ordinate of the lowest zero of each function shown in Table 6.3. note that for $\zeta$ the estimate agrees with the known value $14.1347251417 \ldots$ to within the precision of the computation. The increase in density of zeros with the conductor and degree is apparent in the graphs. Moreover, as the explicit formula is very sensitive to errors in the coefficients, the fact that we see spikes of height 1 for the low zeros indicates that our coefficients were computed correctly. Each graph took a few minutes to generate. 


\begin{tabular}{|lllll|}
\hline$\rho$ & 1 & $2 \mathrm{a}$ & $2 \mathrm{~b}$ & 3 \\
\hline 1 & $1-x$ & $1-x$ & $1-x$ & $1-x$ \\
$\chi$ & $1-x$ & $1+x$ & $1-x$ & $1-x$ \\
$\rho_{4}$ & $(1-x)^{4}$ & $(1-x)^{2}\left(1-x^{2}\right)$ & $\left(1-x^{2}\right)^{2}$ & $(1-x)\left(1-x^{3}\right)$ \\
$\rho_{4}^{\prime}$ & $(1-x)^{4}$ & $(1+x)^{2}\left(1-x^{2}\right)$ & $\left(1-x^{2}\right)^{2}$ & $(1-x)\left(1-x^{3}\right)$ \\
$\rho_{5}$ & $(1-x)^{5}$ & $(1+x)\left(1-x^{2}\right)^{2}$ & $(1-x)\left(1-x^{2}\right)^{2}$ & $\left(1+x+x^{2}\right)\left(1-x^{3}\right)$ \\
$\rho_{5}^{\prime}$ & $(1-x)^{5}$ & $(1-x)\left(1-x^{2}\right)^{2}$ & $(1-x)\left(1-x^{2}\right)^{2}$ & $\left(1+x+x^{2}\right)\left(1-x^{3}\right)$ \\
$\rho_{6}$ & $(1-x)^{6}$ & $\left(1-x^{2}\right)^{3}$ & $(1+x)^{2}\left(1-x^{2}\right)^{2}$ & $\left(1-x^{3}\right)^{2}$ \\
\hline \hline$\rho$ & 4 & 5 & 6 & \\
\hline 1 & $1-x$ & $1-x$ & $1-x$ & \\
$\chi$ & $1+x$ & $1-x$ & $1+x$ & \\
$\rho_{4}$ & $1-x^{4}$ & $1+x+x^{2}+x^{3}+x^{4}$ & $(1+x)\left(1-x^{3}\right)$ & \\
$\rho_{4}^{\prime}$ & $1-x^{4}$ & $1+x+x^{2}+x^{3}+x^{4}$ & $(1-x)\left(1+x^{3}\right)$ & \\
$\rho_{5}$ & $(1-x)\left(1-x^{4}\right)$ & $1-x^{5}$ & $\left(1+x+x^{2}\right)\left(1+x^{3}\right)$ & \\
$\rho_{5}^{\prime}$ & $(1+x)\left(1-x^{4}\right)$ & $1-x^{5}$ & $\left(1-x+x^{2}\right)\left(1-x^{3}\right)$ & \\
$\rho_{6}$ & $\left(1+x^{2}\right)\left(1-x^{4}\right)$ & $(1-x)\left(1-x^{5}\right)$ & $1-x^{6}$ & \\
\hline
\end{tabular}

TABLE $6.2 . S_{5}$ unramified local factors

\begin{tabular}{|r|r|l||r|r|l|}
\hline$\rho$ & conductor & lowest zero & $\rho$ & conductor & lowest zero \\
\hline 1 & 1 & 14.134725142 & $\chi$ & 3 & 8.039737156 \\
$\rho_{4}$ & 4009008 & 1.108937765 & $\rho_{4}^{\prime}$ & 36081072 & 0.5717508665 \\
$\rho_{5}$ & 36081072 & 1.062064850 & $\rho_{5}^{\prime}$ & 12027024 & 0.8132800720 \\
$\rho_{6}$ & 36081072 & 1.376872200 & & & \\
\hline
\end{tabular}

TABLE 6.3. Conductor and ordinate of the lowest zero of each irreducible $L$-function

6.3. Computing $G^{(k)}\left(u_{m} ; \eta,\left\{\mu_{j}\right\}\right)$. Next we compute local approximations of $G\left(u ; \eta,\left\{\mu_{j}\right\}\right)$ for $u$ in the interval $\left[\log \frac{1}{\sqrt{N}}, \log \frac{2^{32}}{\sqrt{N}}\right]$. We evaluate $2^{13}$ Taylor series of 16 terms using (5.9); with these choices, the error term in (5.16) is less than $10^{-28}$. This calculation is the most delicate, due to high precision and catastrophic cancellation. Nevertheless, the computation time for this stage was only a few hours. The graph of $\left|G\left(u ; \eta,\left\{\mu_{j}\right\}\right)\right|$ is shown in Figure 6.2

6.4. Computing $S_{m}^{(k)},(15.18)$ and $L\left(s, \rho_{6}\right)$. Now we come to the main part of the computation, (5.18). Most of the time, approximately twelve hours, was spent computing $S_{m}^{(k)}$. Note that if we had not adjusted $\eta$ to reduce the precision, this calculation could have taken substantially longer.

Once we have $S_{m}^{(k)}$, the computation of (5.18) and $L\left(s, \rho_{6}\right)$ is very fast. We choose $B=\frac{2 \pi \cdot 2^{12}}{\log \left(2^{32}\right)} \approx 1160$. Since this is much larger than $t=115$, the errors terms from Lemma 5.5 are negligible. We choose $A=2^{20} / B \approx 900$, which is about 160 times the expected 

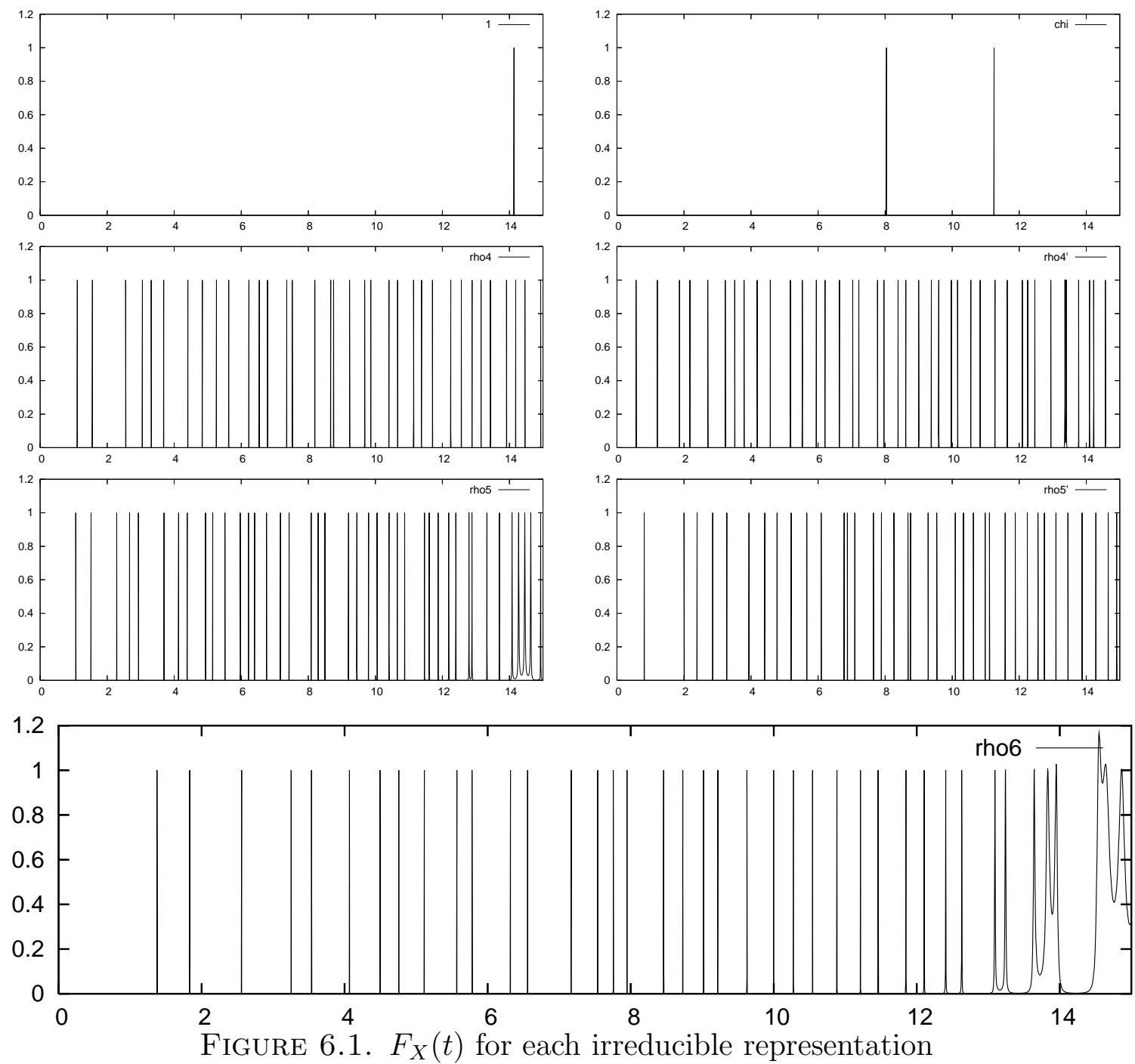

density $\frac{1}{2 \pi} \log N\left(\frac{t}{2 \pi}\right)^{r}$ of zeros around $t=115$. Thus, the main Fourier transform is of $2^{20}$ points, which takes only a few minutes to compute.

Figure 6.3 shows the graph of $Z(t):=\Lambda\left(\frac{1}{2}+i t\right) /\left|\gamma\left(\frac{1}{2}+i t\right)\right|$, which is the analogue of Riemann's $Z$ function. We have superimposed the graph of $25 F_{X}(t)$ over the same range; note the good agreement in location of zeros between the two, which gives evidence that our computations are correct. Figure 6.4 shows $Z(t)$ over the higher range $t \in[90,100]$.

6.5. Turing's method. Finally, we apply Turing's method to the computed $L$-functions. At the same time, we verify the "working hypothesis" that we can isolate the zeros of the irreducible $L$-functions. This verification takes only a few seconds. 

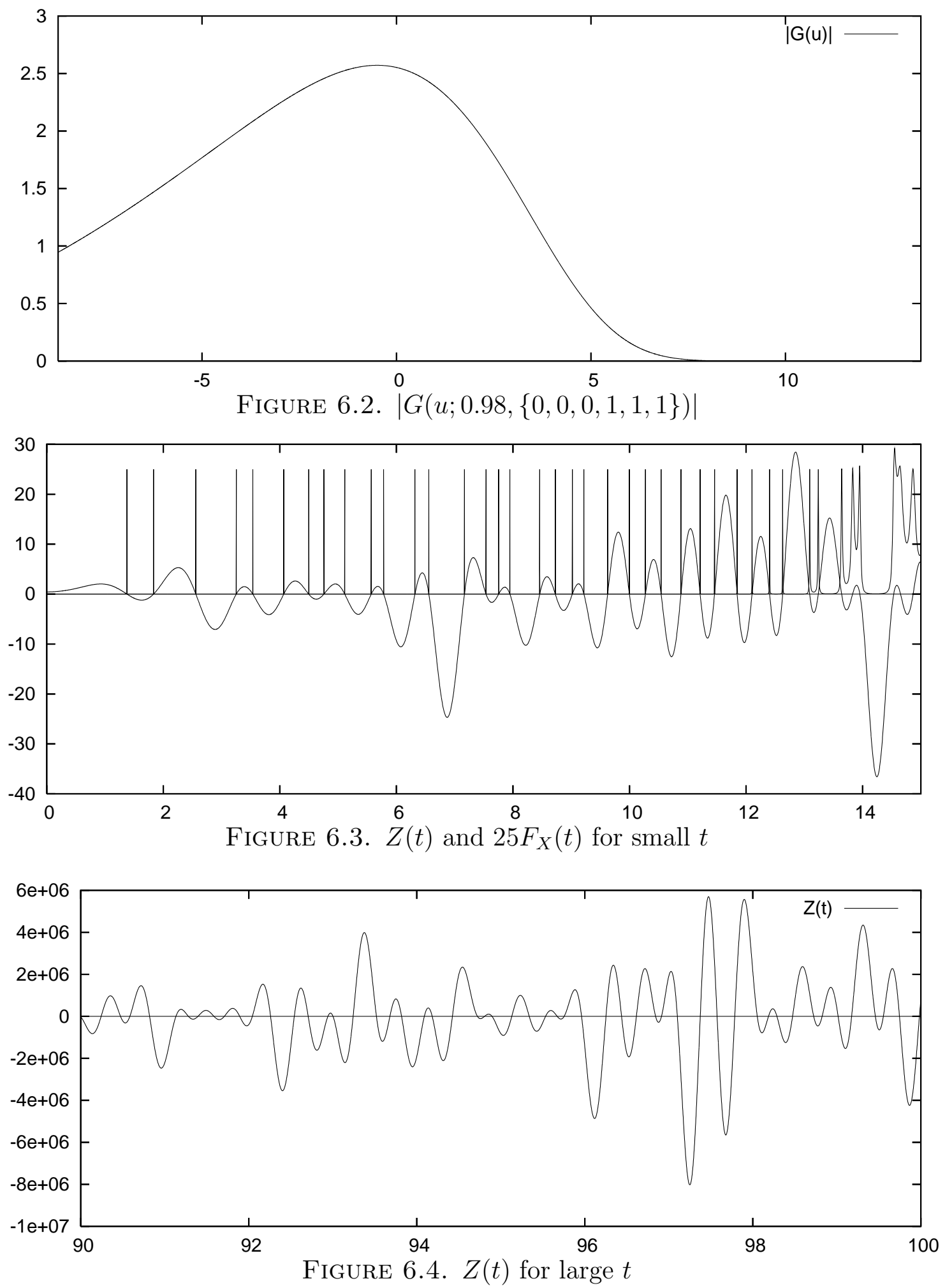


\section{REFERENCES}

[Arm72] J.V. Armitage. Zeta functions with a zero at $s=\frac{1}{2}$. Invent. Math., 16:195-205, 1972.

[Art30] E. Artin. Zur Theorie der L-Reihen mit allgemeinen Gruppencharakteren. Abh. Math. Sem. Univ. Hamburg, 8:292-306, 1930.

[BDSBT01] K. Buzzard, M. Dickinson, N. Shepard-Barron, and R. Taylor. On icosahedral Artin representations. Duke Math. J., 109:283-318, 2001.

[Boo03a] Andrew R. Booker. Numerical tests of modularity. PhD thesis, Princeton University, 2003. available from http://www. umich.edu/ arbooker/papers/.

[Boo03b] Andrew R. Booker. Poles of Artin $L$-functions and the strong Artin conjecture. Ann. of Math. (2), 158(3):1089-1098, 2003.

[Bra47] R. Brauer. On Artin's L-series with general group characters. Ann. Math., 48:502-514, 1947.

[BS02] K. Buzzard and W. Stein. A mod five approach to modularity of icosahedral Galois representations. Pac. Jour. Math., 203(2):265-282, 2002.

[BS05] A. Booker and A. Strömbergsson. Numerical computations with the trace formula and the Selberg eigenvalue conjecture. in preparation, 2005.

[BSV05] A. Booker, A. Strömbergsson, and A. Venkatesh. Effective computation of Maass cusp forms. preprint, 2005.

[Buh78] J.P. Buhler. Icosahedral Galois representations. Springer-Verlag, Berlin, 1978. Lecture Notes in Mathematics, Vol. 654.

[Dok04] Tim Dokchitser. Computing special values of motivic $L$-functions. Experiment. Math., 13(2):137-149, 2004.

[DS74] Pierre Deligne and Jean-Pierre Serre. Formes modulaires de poids 1. Ann. Sci. École Norm. Sup. (4), 7:507-530 (1975), 1974.

[Dum91]Ｄ. S. Dummit. Solving solvable quintics. Math. Comp., 57(195):387-401, 1991.

[Fli94] Yuval Z. Flicker. On the symmetric square: total global comparison. J. Funct. Anal., 122(2):255-278, 1994.

[FM89] Richard Foote and V. Kumar Murty. Zeros and poles of Artin L-series. Math. Proc. Cambridge Philos. Soc., 105(1):5-11, 1989.

[GAP05] The GAP Group. GAP - Groups, Algorithms, and Programming, Version 4.4, 2005. (http://www.gap-system.org).

[IS00] H. Iwaniec and P. Sarnak. Perspectives on the analytic theory of L-functions. Geom. Funct. Anal., (Special Volume, Part II):705-741, 2000. GAFA 2000 (Tel Aviv, 1999).

[JM00] A. Jehanne and M. Müller. Modularity of an odd icosahedral representation. J. Théor. Nombres Bordeaux, 12(2):475-482, 2000. Colloque International de Théorie des Nombres (Talence, 1999).

[JM01] A. Jehanne and M. Müller. Modularity of some odd icosahedral representations. unpublished, 2001. available from http://www.math.u-bordeaux1.fr/ jehanne/travaux.html.

[Kim94] I. Kiming. On the experimental verification of the Artin conjecture for 2-dimensional odd Galois representations over Q. Liftings of 2-dimensional projective Galois representations over Q. In On Artin's conjecture for odd 2-dimensional representations, volume 1585 of Lecture Notes in Math., pages 1-36. Springer, Berlin, 1994.

$[\mathrm{KM}] \quad$ Jürgen Klüners and Gunter Malle. A database for number fields. available from http://www.mathematik. uni-kassel.de/ klueners/minimum/minimum.html.

[Lan80] R.P. Langlands. Base change for GL(2). Princeton University Press, Princeton, N.J., 1980.

[Leh70] R. Sherman Lehman. On the distribution of zeros of the Riemann zeta-function. Proc. London Math. Soc. (3), 20:303-320, 1970.

[LO79] J. C. Lagarias and A. M. Odlyzko. On computing Artin $L$-functions in the critical strip. Math. Comp., 33(147):1081-1095, 1979. 
[LRS99] Wenzhi Luo, Zeév Rudnick, and Peter Sarnak. On the generalized Ramanujan conjecture for GL(n). In Automorphic forms, automorphic representations, and arithmetic (Fort Worth, TX, 1996), volume 66 of Proc. Sympos. Pure Math., pages 301-310. Amer. Math. Soc., Providence, RI, 1999.

[Odl87] A. M. Odlyzko. On the distribution of spacings between zeros of the zeta function. Math. Comp., 48:273-308, 1987.

[Oma01] Sami Omar. Localization of the first zero of the Dedekind zeta function. Math. Comp., 70(236):1607-1616 (electronic), 2001.

[OS88] A. M. Odlyzko and A. Schönhage. Fast algorithms for multiple evaluations of the Riemann zeta function. Trans. Amer. Math. Soc., 309(2):797-809, 1988.

[RR05] Nathalie Revol and Fabrice Rouiller. Multiple precision floating-point interval library, version 1.3.3, 2005. available from http://perso.ens-lyon.fr/nathalie.revol/software.html.

[RS96] Zeév Rudnick and Peter Sarnak. Zeros of principal $L$-functions and random matrix theory. Duke Math. J., 81(2):269-322, 1996. A celebration of John F. Nash, Jr.

[Rub05] Michael Rubinstein. Computational methods and experiments in analytic number theory. preprint, 2005.

[Rum93] Robert Rumely. Numerical computations concerning the ERH. Math. Comp., 61(203):415-440, S17-S23, 1993.

[Tay03] Richard Taylor. On icosahedral Artin representations. II. Amer. J. Math., 125(3):549-566, 2003.

[The04] The PARI Group, Bordeaux. PARI/GP, version 2.1.5, 2004. available from http://pari.math.u-bordeaux.fr/.

[Tit86] E. C. Titchmarsh. The theory of the Riemann zeta-function. The Clarendon Press Oxford University Press, New York, second edition, 1986. Edited and with a preface by D. R. HeathBrown.

[Tol97] Emmanuel Tollis. Zeros of Dedekind zeta functions in the critical strip. Math. Comp., 66(219):1295-1321, 1997.

[Tun81] J. Tunnell. Artin's conjecture for representations of octahedral type. Bull. AMS, 5:173-175, 1981.

[Tur53] A. M. Turing. Some calculations of the Riemann zeta-function. Proc. London Math. Soc. (3), 3:99-117, 1953.

[Wan03] Song Wang. On the symmetric powers of cusp forms on GL(2) of icosahedral type. Int. Math. Res. Not., (44):2373-2390, 2003.

[Wei99] M. Weissman. Icosahedral Galois representations and modular forms. Princeton University, Undergraduate thesis, 1999. available from http://math. berkeley.edu/ marty/.

Mathematics Department, 530 Church Street, University of Michigan, Ann Arbor, Mi 48109

E-mail address: arbooker@umich.edu 